University of New Hampshire

University of New Hampshire Scholars' Repository

Physics Scholarship

Physics

$12-2014$

\title{
Quantifying the relative contributions of substorm injections and chorus waves to the rapid outward extension of electron radiation belt
}

Q. Zong

C A. Kletzing

University of lowa

W. S. Kurth

University of lowa

G. B. Hospodarsky

University of lowa

Harlan E. Spence

University of New Hampshire, harlan.spence@unh.edu

See next page for additional authors

Follow this and additional works at: https://scholars.unh.edu/physics_facpub

Part of the Physics Commons

\section{Recommended Citation}

$\mathrm{Su}, \mathrm{Z}$., et al. (2014), Quantifying the relative contributions of substorm injections and chorus waves to the rapid outward extension of electron radiation belt, J. Geophys. Res. Space Physics, 119, 10,023-10,040, doi:10.1002/2014JA020709.

This Article is brought to you for free and open access by the Physics at University of New Hampshire Scholars' Repository. It has been accepted for inclusion in Physics Scholarship by an authorized administrator of University of New Hampshire Scholars' Repository. For more information, please contact Scholarly.Communication@unh.edu. 


\section{Authors}

Q. Zong, C A. Kletzing, W. S. Kurth, G. B. Hospodarsky, Harlan E. Spence, Geoffrey Reeves, H. O. Funsten, J. B. Blake, and D. N. Baker 


\section{Journal of Geophysical Research: Space Physics}

\section{RESEARCH ARTICLE \\ 10.1002/2014JA020709 \\ Special Section: \\ Quantifying the relative contributions of substorm injections and chorus waves to the rapid outward extension of electron radiation belt}

New perspectives on Earth's radiation belt regions from the prime mission of the Van Allen Probes

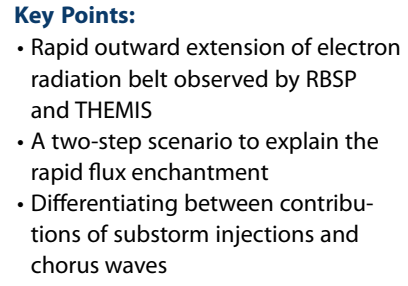

- Rapid outward extension of electron radiation belt observed by RBSP and THEMIS

- A two-step scenario to explain the rapid flux enchantment

- Differentiating between contributions of substorm injections and chorus waves

Correspondence to:

Z. Su,

szpe@mail.ustc.edu.cn

\section{Citation:}

Su, Z., et al. (2014), Quantifying the relative contributions of substorm injections and chorus waves to the rapid outward extension of electron radiation belt, J. Geophys. Res. Space Physics, 119, 10,023-10,040, doi:10.1002/2014JA020709.

Received 8 OCT 2014

Accepted 26 NOV 2014

Accepted article online 2 DEC 2014

Published online 19 DEC 2014

Abstract We study the rapid outward extension of the electron radiation belt on a timescale of injections and chorus waves to the electron flux enhancement near the outer boundary of radiation belt. A comprehensive analysis including both observations and simulations is performed for the first event on 26 May 2013. The outer boundary of electron radiation belt moved from $L=5.5$ to $L>6.07$ over about $6 \mathrm{~h}$, with up to 4 orders of magnitude enhancement in the $30 \mathrm{keV}$ to $5 \mathrm{MeV}$ electron fluxes at $L=6$. The observations show that the substorm injection can cause $100 \%$ and $20 \%$ of the total subrelativistic $(\sim 0.1 \mathrm{MeV})$ and relativistic (2-5 MeV) electron flux enhancements within a few minutes. The data-driven simulation supports that the strong chorus waves can yield $60 \%-80 \%$ of the total energetic $(0.2-5.0 \mathrm{MeV})$

\begin{abstract}
Zhenpeng Su',2, Hui Zhu' ${ }^{1,2,3}$, Fuliang Xiao ${ }^{4}$, Huinan Zheng ${ }^{1,2}$, Yuming Wang ${ }^{1}$, Q.-G. Zong ${ }^{5}$, Zhaoguo $\mathrm{He}^{6}$, Chao Shen ${ }^{7}$, Min Zhang ${ }^{1}$, Shui Wang ${ }^{1}$, C. A. Kletzing ${ }^{8}$, W. S. Kurth ${ }^{8}$, G. B. Hospodarsky ${ }^{8}$, H. E. Spence ${ }^{9}$, G. D. Reeves ${ }^{10}$, H. O. Funsten ${ }^{11}$, J. B. Blake ${ }^{12}$, and D. N. Baker ${ }^{13}$

${ }^{1}$ CAS Key Laboratory of Geospace Environment, Department of Geophysics and Planetary Sciences, University of Science and Technology of China, Hefei, China, ${ }^{2}$ State Key Laboratory of Space Weather, Chinese Academy of Sciences, Beijing, China, ${ }^{3}$ Mengcheng National Geophysical Observatory, School of Earth and Space Sciences, University of Science and Technology of China, Hefei, China, ${ }^{4}$ School of Physics and Electronic Sciences, Changsha University of Science and Technology, Changsha, China, ${ }^{5}$ Institute of Space Physics and Applied Technology, Peking University, Beijing, China, ${ }^{6}$ Center for Space Science and Applied Research, Chinese Academy of Sciences, Beijing, China, ${ }^{7}$ State Key Laboratory of Space Weather, Center for Space Science and Applied Research, Chinese Academy of Sciences, Beijing, China,

${ }^{8}$ Department of Physics and Astronomy, University of lowa, lowa City, lowa, USA, ${ }^{9}$ Institute for the Study of Earth, Oceans, and Space, University of New Hampshire, Durham, New Hampshire, USA, ${ }^{10}$ Space Science and Applications Group, Los Alamos National Laboratory, Los Alamos, New Mexico, USA, ${ }^{11}$ ISR Division, Los Alamos National Laboratory, Los Alamos, New Mexico, USA, ${ }^{12}$ The Aerospace Corporation, Los Angeles, California, USA, ${ }^{13}$ Laboratory for Atmospheric and Space Physics, University of Colorado Boulder, Boulder, Colorado, USA
\end{abstract} several hours during three events observed by Radiation Belt Storm Probes and Time History of Events and Macroscale Interactions during Substorms satellites and particularly quantify the contributions of substorm electron flux enhancement within about $6 \mathrm{~h}$. Some simple analyses are further given for the other two events on 2 and 29 June 2013, in which the contributions of substorm injections and chorus waves are shown to be qualitatively comparable to those for the first event. These results clearly illustrate the respective importance of substorm injections and chorus waves for the evolution of radiation belt electrons at different energies on a relatively short timescale.

\section{Introduction}

The electron radiation belts exhibit dramatic variability over timescales from minutes to decades [e.g., Blake et al., 1992; Hudson et al., 1997; Reeves et al., 1998; Li et al., 2001; Horne et al., 2005b; Su et al., 2011a; Baker et al., 2013b; Shprits et al., 2013]. These complex radiation belt dynamics can be attributed to the competition and/or cooperation of various acceleration, loss, and transport mechanisms [Reeves et al., 2003]. One important area of research is to differentiate among the contributions of those physical mechanisms to the radiation belt evolution. Here we concentrate on two processes for the radiation belt evolution on a timescale of hours: earthward injection during substorms and local acceleration by whistler mode chorus waves.

Substorm injection is a large-scale transport process from the magnetotail to the inner magnetosphere associated with the dipolarization of the geomagnetic field. It usually causes the enhancement of electron fluxes over a wide range of energies from tens to hundreds of $k \mathrm{kV}$ in the region $L=4-8$ on a timescale of minutes [e.g., Reeves et al., 1990; Friedel et al., 1996; Baker et al., 1997, 1998]. 
These substorm-injected subrelativistic electrons can serve as the seed particles for the subsequent acceleration to relativistic energies [Hwang et al., 2004]. Through the test particle simulations, Liu et al. [2003] showed that the quiescent Stern-Volland convection electric fields [Stern, 1973; Volland, 1973] cannot inject the relativistic electrons into the radiation belt region. Li et al. [1998, 2003] emphasized the importance of substorm-associated impulsive electric fields for the injection of energetic electrons. In these test particle simulations [Li et al., 1998, 2003], the initial energy of midtail electrons and the impulsive electric fields appeared to be too weak to produce the injection of relativistic electrons. Based on the phase space density analysis, Lui et al. [2012] suggested that the source electrons in the region $L>8$ during dipolarization intervals are adequate to populate the outer radiation belt even in the relativistic energy range. Based on the observations of several radially displaced satellites, Dai et al. [2014] tracked the substorm transport process from the magnetotail to the geostationary orbit and found the injection of relativistic (up to several $\mathrm{MeV}$ ) electrons by the dipolarization-associated intense electric fields.

Whistler mode chorus waves are often observed in the plasma trough region during geomagnetically disturbed periods [e.g., Meredith et al., 2001], which are excited by the anisotropic suprathermal (from a few keV to tens of keV) electrons near the magnetic equator [e.g., Omura et al., 2008; Li et al., 2009]. The chorus emissions typically split into lower and upper bands with a gap around $0.5 f_{\text {ce }}$ ( $f_{\text {ce }}$ is the equatorial electron gyrofrequency) [e.g., Tsurutani and Smith, 1974; Santolík et al., 2003a]. The theoretical [Horne and Thorne, 1998; Summers et al., 1998], observational [e.g., Horne et al., 2005a; Meredith et al., 2012; Agapitov et al., 2013; Thorne et al., 2013; Mourenas et al., 2014; Su et al., 2014b], and numerical [e.g., Summers et al., 2002; Li et al., 2005; Glauert and Horne, 2005; Shprits et al., 2006; Albert et al., 2009; Su et al., 2010; Mourenas et al., 2012; Glauert et al., 2014] works have revealed the importance of chorus-driven local acceleration for the enhancement of radiation belt relativistic electrons on a timescale of days.

In this study, we investigate the rapid outward extension process of the electron radiation belt within several hours for three events observed by the Radiation Belt Storm Probes (RBSP) [Mauk et al., 2013] and the Time History of Events and Macroscale Interactions during Substorms (THEMIS) satellites [Angelopoulos, 2008]. For the first event on 26 May 2013, a comprehensive analysis is performed to quantitatively determine the respective contributions of substorm injection and chorus waves to the electron flux enhancement around the outer boundary of radiation belt. The generality of obtained results is further examined in the other two events on 2 and 29 June 2013.

\section{Rapid Outward Extension}

2.1. Event on 26 May 2013

2.1.1. Observations

Figure 1 plots the interplanetary and magnetospheric parameters during 24-27 May 2013 provided by the CDAweb-OMNI database. A series of interacting solar ejecta carrying intermittent southward magnetic fields and multiple shocks successively hit the Earth's magnetosphere. Consequently, a multistep geomagnetic storm (with the minimum SYM- $H$ value of $-65 \mathrm{nT}$ and the maximum $K_{p}$ value of 5 ) and prolonged substorm activity (with the maximum $A E$ value of $1700 \mathrm{nT}$ ) were triggered. Our study primarily covers the time range 05:20-12:40 UT on 26 May 2013 (indicated by the yellow shadow), corresponding to the late recovery phase of this geomagnetic storm. In this time range, the SYM- $H$ index fluctuated around $-40 \mathrm{nT}$, implying the insignificant variation of magnetic field topology associated with the ring current development. Note that the data gaps in the interplanetary parameters do not affect our following analysis.

Figure 2 shows the spin-averaged electron fluxes observed by the Magnetic Electron Ion Spectrometer (MagEIS) [Blake et al., 2013] and Relativistic Electron-Proton Telescope (REPT) [Baker et al., 2013a] of the Energetic Particle, Composition, and Thermal Plasma (ECT) suite [Spence et al., 2013] on board the twin RBSP satellites. The two RBSP satellites were in nearly identical orbit (period $\sim 9 \mathrm{~h}$ ) but separated by about $1 \mathrm{~h}$ of local time. The electron fluxes around the outer boundary of radiation belt are further plotted in Figure 3. In the inbound pass of RBSP-B (Figure 3a), the satellite encountered the outer boundary of radiation belt at 06:00 UT $(L \approx 5.5)$ and the substorm injection front at 06:14 UT $(L \approx 5.0$ ). In the inbound pass of RBSP-A (Figure $3 \mathrm{~b}$ ), the satellite found the substorm injection front at 06:12 UT ( $L \approx 6.4)$. The dispersionless characteristic of substorm injection [Mcllwain, 1974] can be clearly identified by RBSP-A satellite around midnight. Since the substorm injection propagated from the magnetotail to the radiation belt region, the RBSP-A satellite at the outer region detected the injection front approximately 2 min ahead of the RBSP-B satellite in the inner region. Thus, based on the time-distance 

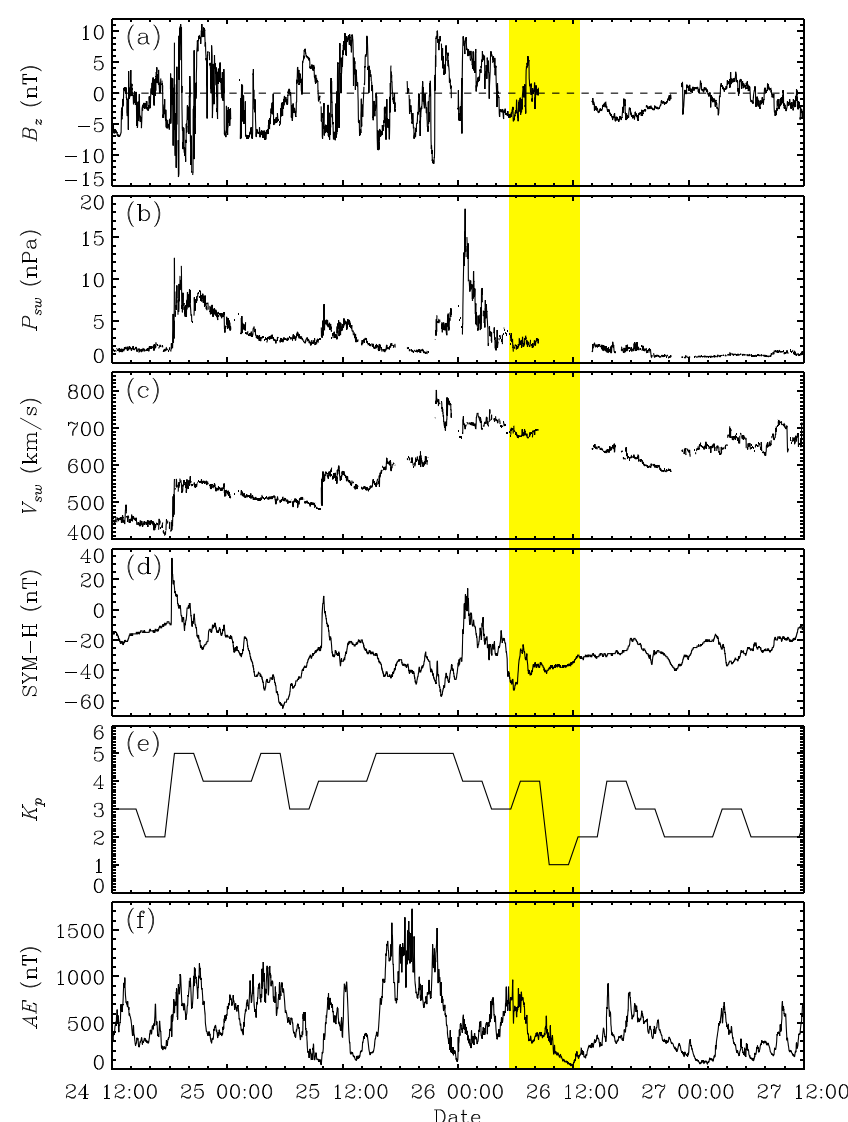

Figure 1. Interplanetary and magnetospheric parameters during 24-26 May 2013: (a) interplanetary magnetic field in the GSE coordinate system $B_{z} ;\left(\right.$ b) solar wind dynamic pressure $P_{\text {sw }} ;$ (c) solar wind speed $V_{\text {swi }}$ (d-f) geomagnetic activity indices $S Y M-H, K_{p}$, and $A E$. The yellow shadow denotes the time range of interest from 05:20 UT to 12:40 UT on 26 May 2013.

relationship, the corresponding propagation speed $V_{s}$ of substorm injection may be obtained $V_{s} \approx 74 \mathrm{~km} / \mathrm{s}$, comparable to the estimations in the early works [Russell and McPherron, 1973; Moore et al., 1981].

Throughout the outbound pass of RBSP-B (Figure 3c), the satellite collected the smoothly varying (rather than fluctuating) electron fluxes, indicating that the electron radiation belt had extended at least beyond $L=6.07$.

Figure 4 exhibits the VLF/ELF wave characteristics observed by the Electric and Magnetic Field Instrument Suite and Integrated Science (EMFISIS) instrument [Kletzing et al., 2013] on board the RBSP satellites and derived from the filter bank data [Bonnell et al., 2008] of the THEMIS-D spacecraft. The annotated frequencies $0.05 f_{\text {ce }}$ and $0.50 f_{\text {ce }}$ are estimated from the geomagnetic field model (International Geomagnetic Reference Field (IGRF) model for the internal field and Olson-Pfitzer (OP77) model [Olson and Pfitzer, 1982] for the external field) for the two RBSP satellites and calculated from the local magnetic field measurements for the THEMIS-D satellite. Following the substorm injection, the two RBSP satellites found strong lower band chorus waves (with power spectral density $B_{f}^{2}$ up to $10^{-4} \mathrm{nT}^{2} / \mathrm{Hz}$ ) in the postmidnight plasma trough, intense hiss waves in the plasmasphere, but no obvious wave activity in the premidnight plasma trough. Benefiting from the special orbit, the THEMIS-D satellite continuously detected the strong lower band chorus waves (with wave amplitude $B_{t}$ up to $\left.0.2 \mathrm{nT}\right)$ in the equatorial plasma trough $(5.5<L<9.9)$. These observed distribution characteristics of VLF/ELF waves are roughly consistent with the previous statistical results [e.g., Meredith et al., 2001].

Figure 5 presents a snapshot of VLF/ELF waveforms recorded by EMFISIS instrument of RBSP-B around 06:22:18 UT. The fast Fourier transform was used on the waveform data to obtain the electromagnetic spectral matrices and the corresponding electric and magnetic power spectral densities. Subsequently, the singular value decomposition method [Santolik et al., 2003b] was adopted to analyze the normal angle and 


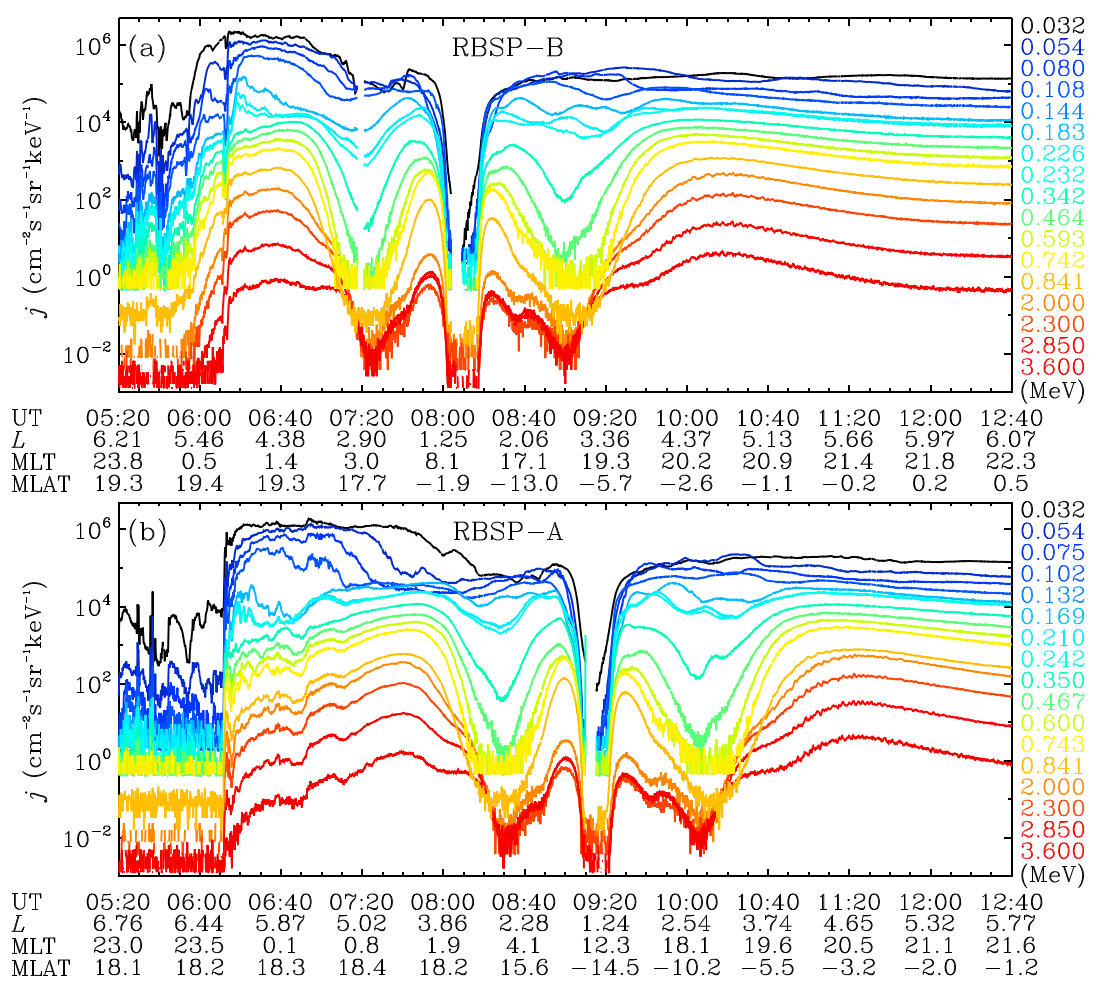

Figure 2. Evolution of the radiation belt spin-averaged electron fluxes at different energies $E_{k}=32 \mathrm{keV}$ to $3.6 \mathrm{MeV}$ observed by (a) RBSP-B and (b) RBSP-A on 26 May 2013.

ellipticity. The discrete rising tones can be clearly identified in the magnetic and electric power spectral densities. These waves propagated in a quasi field-aligned direction (mostly with the normal angles $\theta<30^{\circ}$ ) and were predominantly circularly polarized (with ellipticity close to 1). These observed characteristics fit the typical descriptions of chorus waves in the plasma trough region [e.g., Santolík et al., 2003a, 2014b].

Figure 6 gives the ULF wave characteristics observed by the EMFISIS instrument of the RBSP satellites in the outer belt region. The wavelet transform [Grinsted et al., 2004] has been performed on the observed magnetic fields to obtain the ULF wave power spectral density [Zong et al., 2009]. The overall trend of ULF waves appeared to be roughly consistent with that of the $K_{p}$ index (see Figure 1e). The ULF waves showed a transient enhancement following the substorm injection $\left(K_{p}=4\right)$ but then kept a relatively low level of intensity especially during the outbound passes of RBSP satellites $\left(K_{p}=1\right)$.

Based on the observations above, we propose a physical scenario to explain the rapid outward extension of electron radiation belt. The first step is the substorm injection of electrons over a wide energy range, and the second step is the further acceleration of electrons by chorus waves (probably excited by injected suprathermal electrons). For example, at $L=6.0$, the substorm injection directly increased the $31 \mathrm{keV} \leq$ $E_{k} \leq 3.6 \mathrm{MeV}$ electron fluxes by $2-4$ orders of magnitude within a few minutes (the magnetotail electrons can be accelerated to high energies in the course of injection), and then the chorus waves enhanced the $0.2 \mathrm{MeV} \leq E_{k} \leq 3.6 \mathrm{MeV}$ electron fluxes by a factor of $2-5$ in the following $\sim 6 \mathrm{~h}$ (see Figure 3 ). The first step is quite evident in the observations, while the second step needs to be examined by the simulations.

It should be mentioned that the storm time buildup of electron fluxes at the center of outer radiation belt on a timescale of days had been explained by a three-step scenario [e.g., Baker et al., 1997, 1998; Reeves et al., 1998; Miyoshi et al., 2003; Shprits et al., 2009b], i.e., the electrons are first injected by substorms then accelerated locally and diffused radially inward and/or outward. Here the rapid outward extension of electron radiation belt occurred on a timescale of several hours, much shorter than that of radial diffusion ( days). In the time range of interest, the $K_{p}$ index was small (see Figure 1e), and the RBSP-observed ULF waves had a low level of intensity most of the time (see Figure 6). The strength of radial diffusion (evaluated from the $K_{p}$-dependent expressions [Brautigam and Albert, 2000]) may be relatively weak, and a very limited contribution of radial diffusion to this rapid extension event should be expected. 


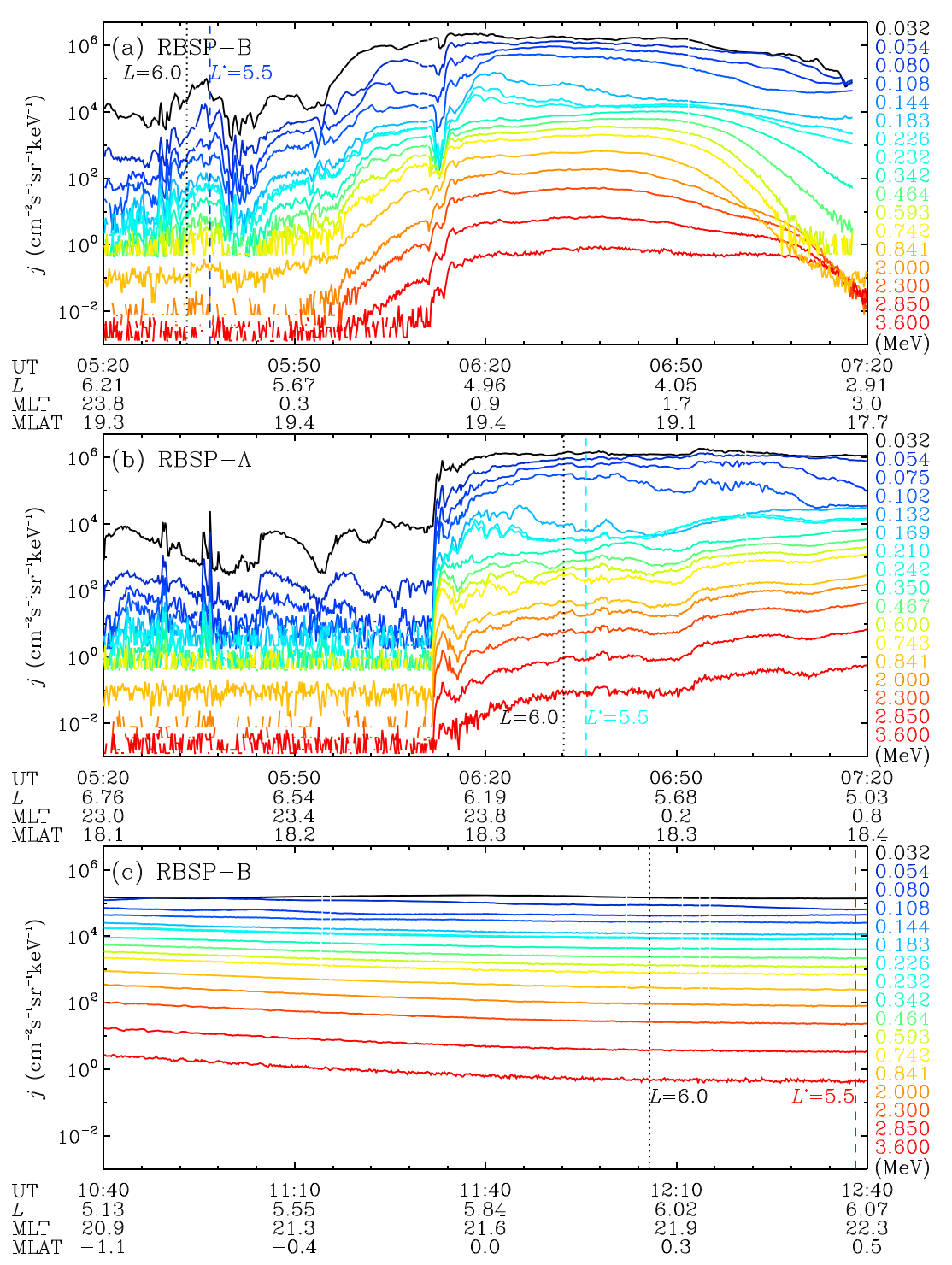

Figure 3. Spin-averaged electron fluxes around the outer boundary of radiation belt on 26 May 2013: (a) RBSP-B observations from 05:20 UT to 07:20 UT; (b) RBSP-A observations from 05:20 UT to 07:20 UT; and (c) RBSP-B observations from 10:40 UT to 12:40 UT. The vertical dotted and dashed lines represent the locations $L=6$ and $L^{*}=5.5$, respectively.

\subsubsection{Simulations}

The chorus-driven electron acceleration process is investigated at a fixed Roederer $L^{*}=5.5$ (close to $L=6.0$ ) based on the two-dimensional storm-time evolution of electron radiation belt (STEERB) code [Xiao et al., 2009; Su et al., 2010, 2014b]. The Roederer L* (provided by the Level 3 data of RBSP) is determined in a geomagnetic field model with the IGRF internal field and OP77 external field. The basic equation for the evolution of electron phase space density (PSD) $F$ is given by

$$
\frac{\partial F}{\partial t}=\frac{1}{G} \frac{\partial}{\partial \alpha_{e}}\left[G\left(\left\langle D_{\alpha \alpha}\right\rangle \frac{\partial F}{\partial \alpha_{e}}+\left\langle D_{\alpha p}\right\rangle \frac{\partial F}{\partial p}\right)\right]+\frac{1}{G} \frac{\partial}{\partial p}\left[G\left(\left\langle D_{p \alpha}\right\rangle \frac{\partial F}{\partial \alpha_{e}}+\left\langle D_{p p}\right\rangle \frac{\partial F}{\partial p}\right)\right]-\frac{F}{\tau_{L}},
$$

with

$$
\begin{aligned}
& G=p^{2} T\left(\alpha_{e}\right) \sin \alpha_{e} \cos \alpha_{e}, \\
& T\left(\alpha_{e}\right) \approx 1.30-0.56 \sin \alpha_{e} .
\end{aligned}
$$

The wave-particle interaction efficiency is characterized by the drift-averaged diffusion coefficients $\left\langle D_{\alpha \alpha}\right\rangle$, $\left\langle D_{p p}\right\rangle$ and $\left\langle D_{\alpha p}\right\rangle=\left\langle D_{p \alpha}\right\rangle$ in the equatorial pitch angle $\alpha_{e}$, momentum $p$, and mixed terms. The electron 

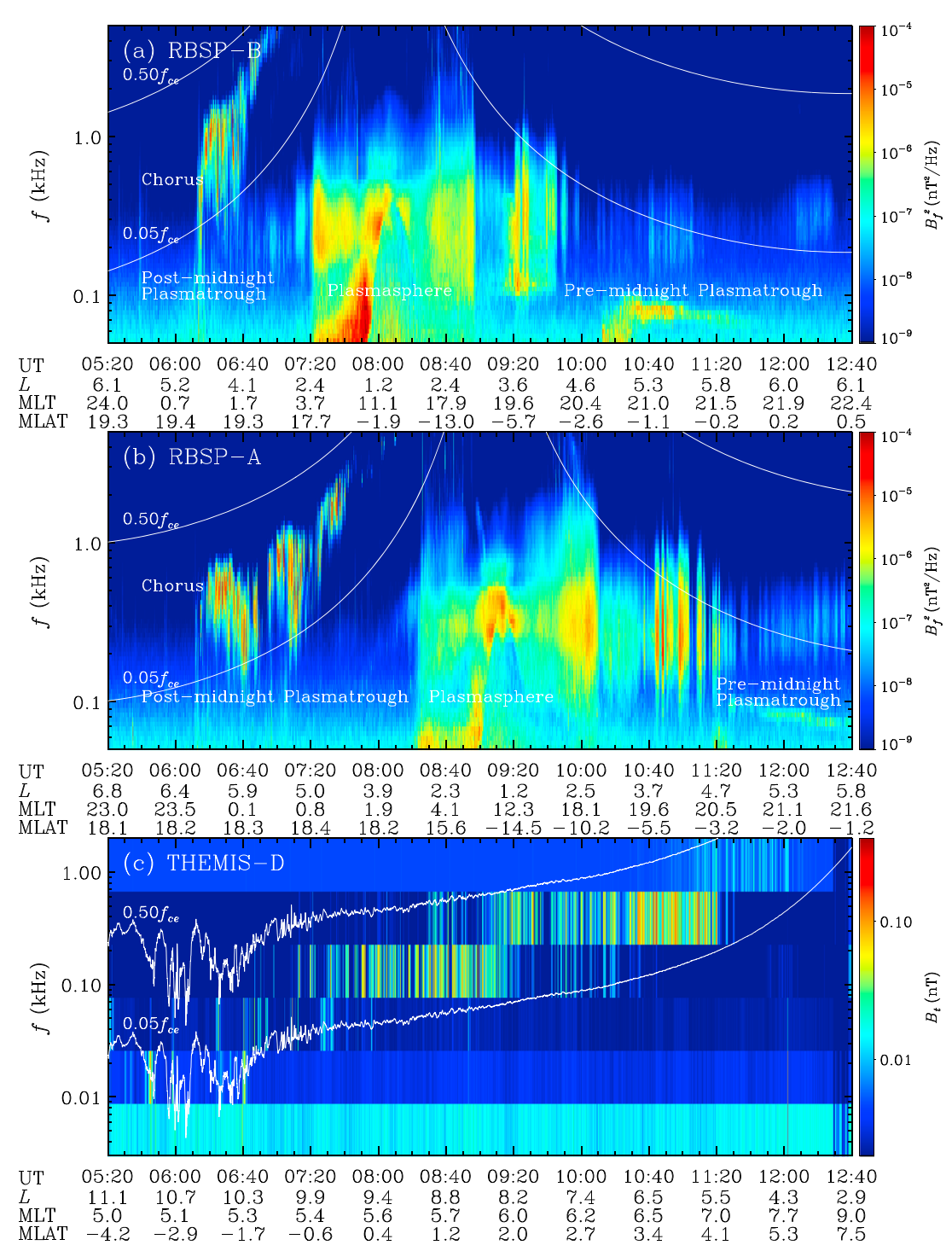

Figure 4. VLF/ELF waves for the 26 May 2013 event: (a and b) wave magnetic power spectral density $B_{f}^{2}$ observed by the twin RBSP satellites and (c) wave magnetic amplitude $B_{t}$ observed by the THEMIS satellite. The white lines represent the frequencies $0.05 f_{\text {ce }}$ and $0.50 f_{\text {ce. }}$.

precipitation in the loss cone is represented by the last term $-F / \tau_{L}$. The empirical lifetime $\tau_{L}$ is set to be a quarter of bounce period in the loss cone (in view of the four atmospheric crossings in one bounce period) [Selesnick et al., 2003; Shprits et al., 2009a] and be infinite out of the loss cone.

These diffusion coefficients are evaluated in the dipole geomagnetic field and controlled by the wave distribution in frequency, normal angle, and space, as well as the ratio between the plasma frequency $f_{\mathrm{pe}}$ and equatorial electron gyrofrequency $f_{\mathrm{ce}}$. Figure $7 \mathrm{a}$ demonstrates the frequency-dependent wave magnetic power spectral densities (circles) observed by RBSP-A around $L=6.0$ and the modeled Gaussian frequency distribution (line)

$$
B_{f}^{2}=\frac{2 B_{t}^{2}}{\pi^{1 / 2} \Delta f}\left[\operatorname{erf}\left(\frac{f_{2}-f_{m}}{\delta f}\right)+\operatorname{erf}\left(\frac{f_{m}-f_{1}}{\delta f}\right)\right]^{-1} \exp \left[-\left(\frac{f-f_{m}}{\Delta f}\right)^{2}\right]
$$

with an amplitude $B_{t}=0.147 \mathrm{nT}$, a central frequency $f_{m}=0.156 f_{\text {ce, }}$, half width $\Delta f=0.029 f_{\text {ce, }}$ a lower limit $f_{1}=0.050 f_{\mathrm{ce}}$, and an upper limit $f_{2}=0.500 f_{\mathrm{ce}}$. The obtained wave amplitude is comparable to that provided by the THEMIS-D satellite (see Figure 4c). The waveform data of RBSP-A are not available in the time range of interest, and the normal angle distribution of chorus waves is obtained from the waveform 


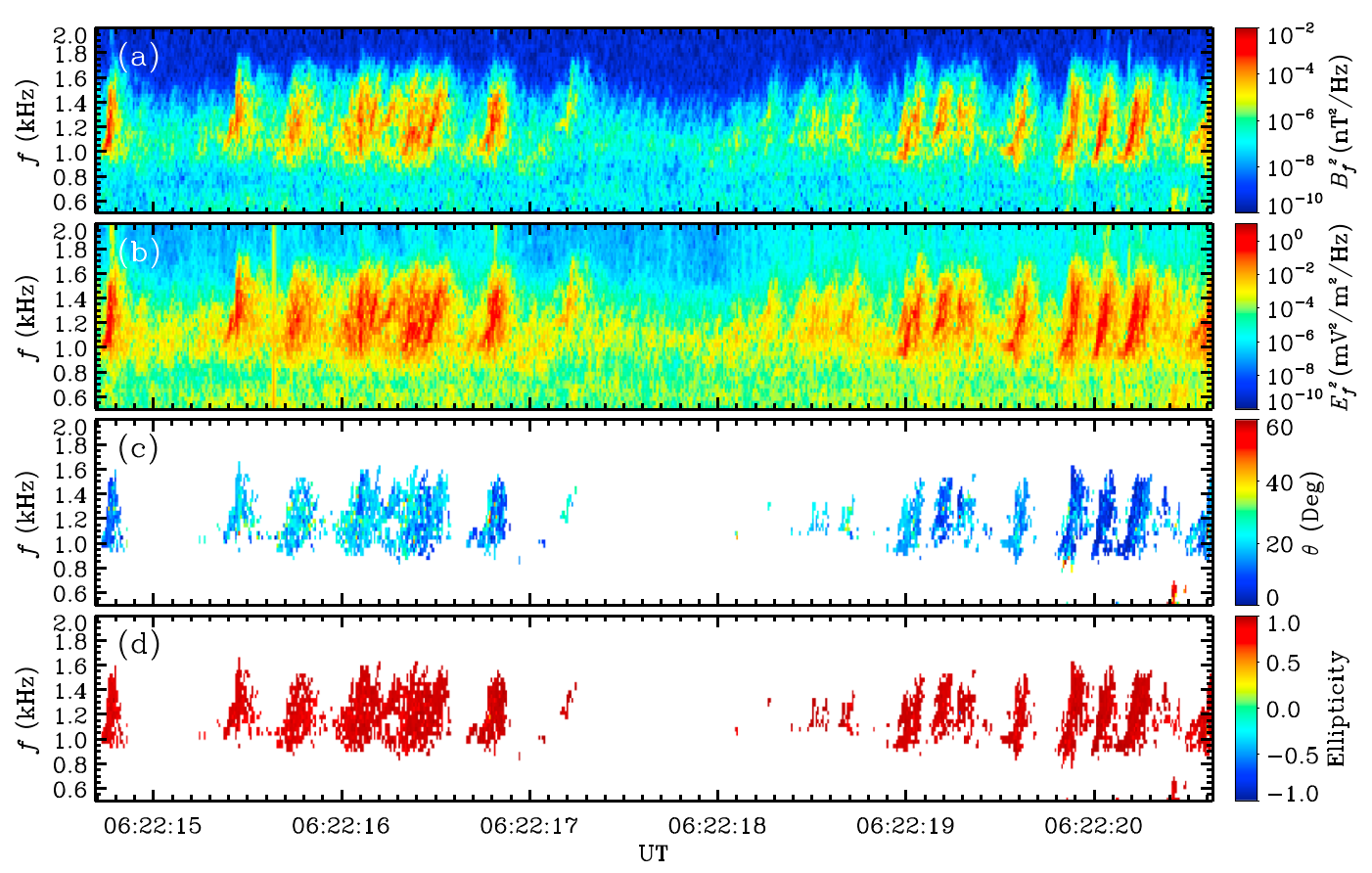

Figure 5. A snapshot of VLF/ELF waveforms recorded by RBSP-B around 06:22:18 UT on 26 May 2013: (a) magnetic power spectral density $B_{f}^{2}$; (b) electric power spectral density $E_{f}^{2}$; (c) wave normal angle $\theta$; and (d) ellipticity of the magnetic field polarization. Note that the normal angle and ellipticity are shown for the waves with power spectral densities $B_{f}^{2}>5 \times 10^{-6} \mathrm{nT}^{2} \mathrm{~Hz}$.
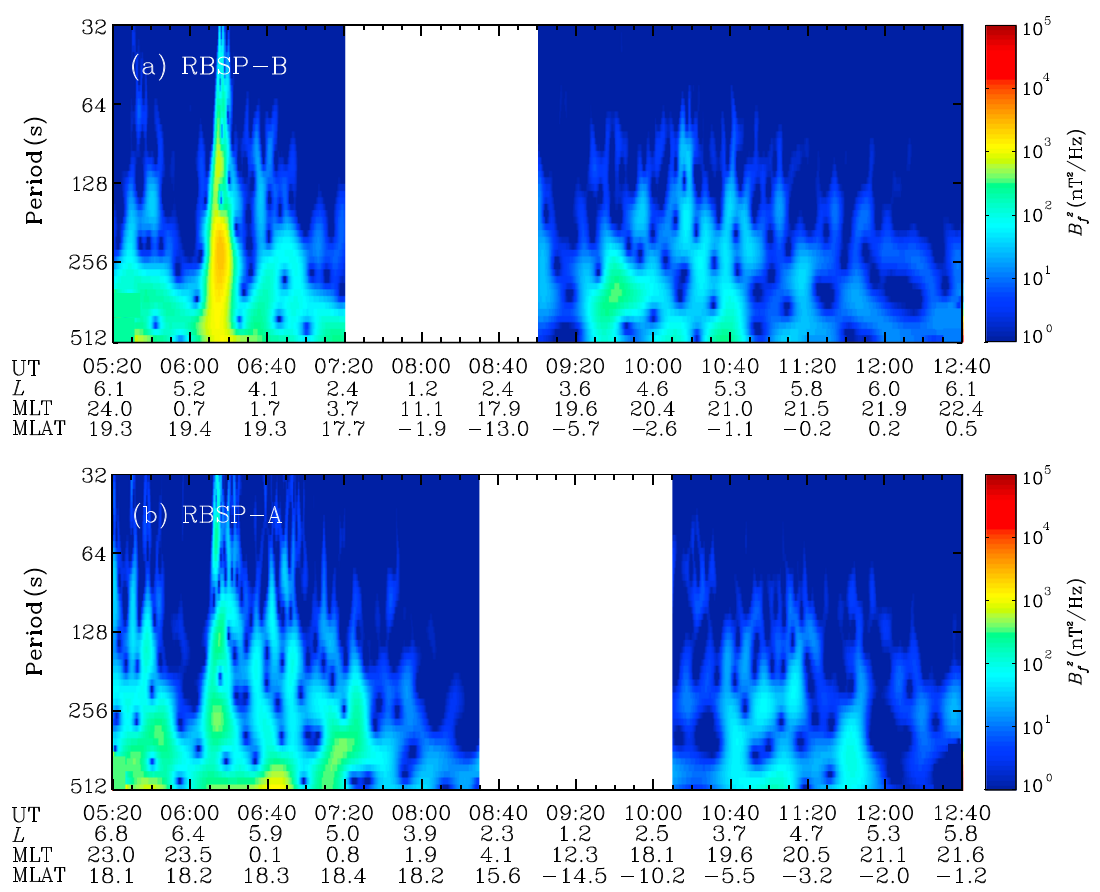

Figure 6. ULF waves for the 26 May 2013 event: wavelet power spectrums of magnetic field observed by (a) RBSP-B and (b) RBSP-A. Note that the blank regions correspond to the inner radiation belt. 

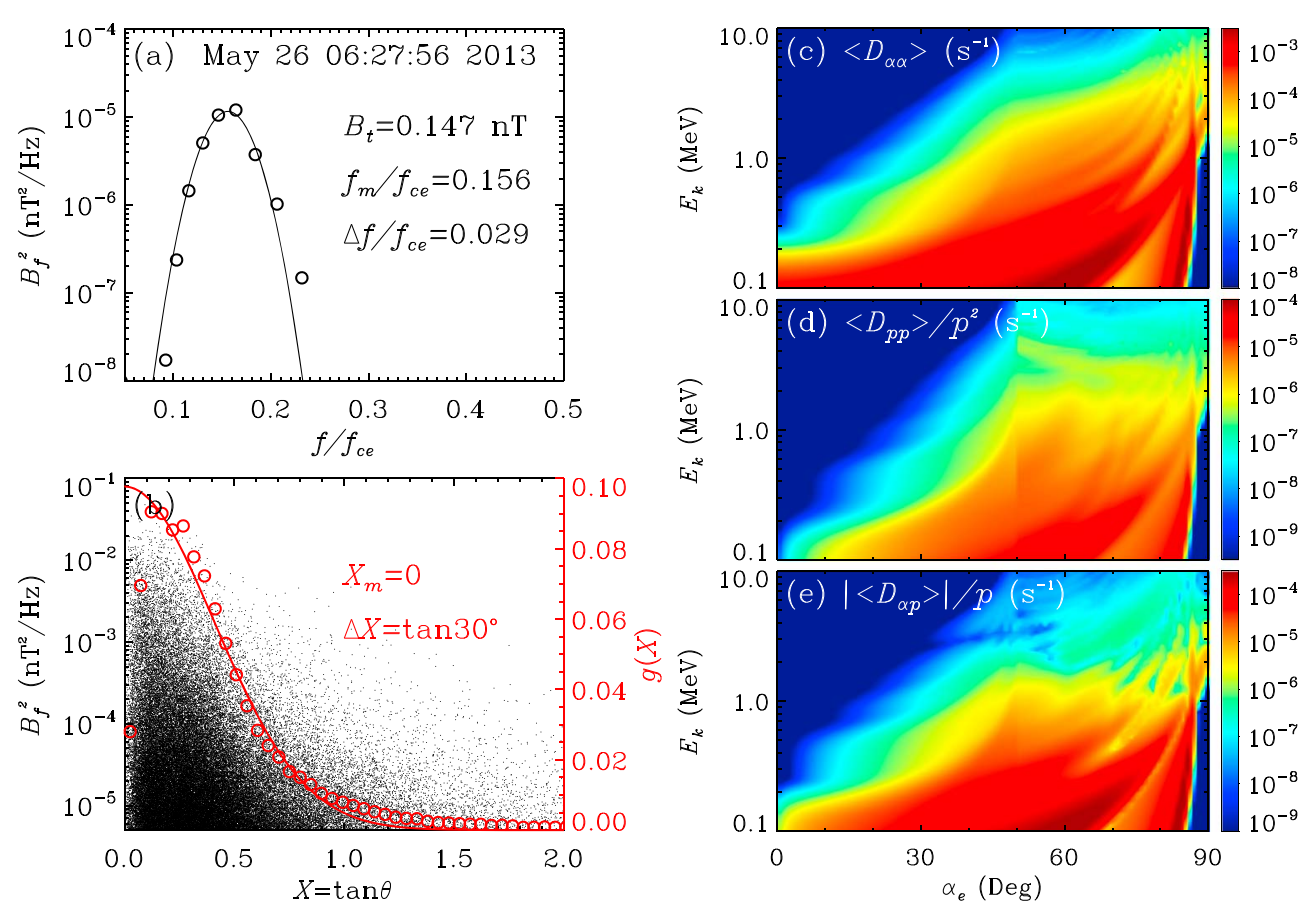

Figure 7. Wave characteristics and quasi-linear diffusion coefficients: (a) observed (circles) and modeled (line) frequency-dependent magnetic power spectral density $B_{f}^{2}$ around $L=6.0$; (b) normal angle-dependent magnetic power spectral density $B_{f}^{2}$ (dots) in the time range from 06:00 UT to 07:00 UT and calculated (circles) and modeled (line) occurrence rates of chorus waves in separate normal angle bins; and (c-e) drift-averaged diffusion coefficients as functions of pitch angle $\alpha_{e}$ and kinetic energy $E_{k}$.

data of RBSP-B. Figure 7b shows the normal angle-dependent magnetic power spectral density $B_{f}^{2}$ (dots) observed by RBSP-B in the time range from 06:00 UT to 07:00 UT, the calculated occurrence rates $g(X=\tan \theta)$ of chorus waves (circles) in separate normal angle bins (with width $\Delta X=0.05$ ), and the modeled Gaussian normal angle distribution (line)

$$
g(\tan \theta) \propto \exp \left[-\left(\frac{\tan \theta-\tan \theta_{m}}{\tan \Delta \theta}\right)^{2}\right]
$$

with a center $\theta_{m}=0^{\circ}$, a half width $\Delta \theta=30^{\circ}$, a lower cutoff $\theta_{1}=0^{\circ}$, and an upper cutoff $\theta_{2}=45^{\circ}$ (the occurrence rate in the normal angles $\theta>45^{\circ}$ were quite small). The observed occurrence rates around $\theta=0^{\circ}$ tended to zero, primarily due to the drastically reduced solid angle around $\theta=0^{\circ}$. These chorus waves are assumed to distribute over a wide range of latitudes $|\lambda| \leq 20^{\circ}$ and magnetic local times MLT $=23.8-7.0$ (see Figures $4 \mathrm{~b}$ and $4 c$ ). In these regions, the wave characteristics and the cold electron density are assumed to be unchanged. The ratio $f_{\text {pe }} / f_{\text {ce }}$ is set to be 6.2 based on the dipole magnetic field model and the electron density model of Sheeley et al. [2001], comparable to that derived from the real-time measurements of upper hybrid frequency [Kletzing et al., 2013] and magnetic field. The distributions of obtained diffusion coefficients in the range of $0^{\circ} \leq \alpha_{e} \leq 90^{\circ}$ and $0.1 \mathrm{MeV} \leq E_{k} \leq 10.0 \mathrm{MeV}$ are plotted in Figures 7c-7e. All the diffusion coefficients in the high-energy range maximize at the relatively large equatorial pitch angles, implying a significant acceleration effect on the trapped electrons.

It should be noted that the specific values of wave parameters can change to some extent in the different events. Here the amplitude $B_{t}=0.147 \mathrm{nT}$ is larger than that $\left(B_{t}=0.05 \mathrm{nT}\right)$ from statistical analysis [e.g., Horne et al., 2005a], smaller than that $\left(B_{t}=0.5-3 \mathrm{nT}\right)$ in some extreme event [e.g., Cattell et al., 2008; Santolík et al., 2014a; Su et al., 2014a], but generally comparable to that $\left(B_{t}=0.1-0.2 \mathrm{nT}\right)$ for some recent event [e.g., Thorne et al., 2013; Li et al., 2014; Xiao et al., 2014]. The central frequency $f_{m} / f_{\text {ce }}=0.156$ is slightly smaller than that $\left(f_{m} / f_{\mathrm{ce}}=0.20-0.35\right)$ in some previous works [e.g., Horne et al., 2005a; Thorne et al., 2013; Li et al., 2014; Xiao et al., 2014] but approximately consistent with that $\left(f_{m} / f_{\text {ce }}=0.165\right)$ in our recent work [Su et al., 2014a]. The acceleration by quasi-parallel chorus $\left(\theta_{m}=0^{\circ}\right)$ has been widely investigated in the previous simulations [e.g., Horne et al., 2005a; Albert et al., 2009; Thorne et al., 2013; Li et al., 2014; Su et al., 2014a; 


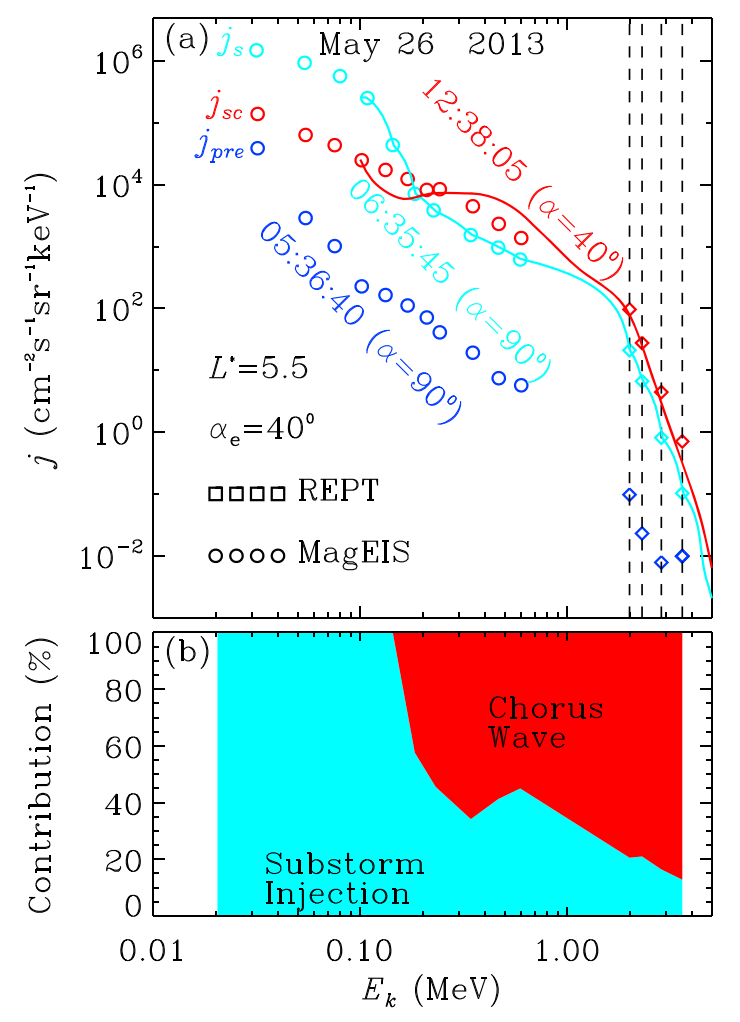

Figure 8. (a) Observed (symbols) and simulated (lines) differential energy spectrums $j=p^{2} F$ at three different time points and (b) the contribution percentages of chorus wave and substorm injection to the total enhancement of energetic electron fluxes. In Figure 8a, the circles and squares represent the observations of MagEIS and REPT instruments, and the vertical dashed lines are plotted to clearly identify the electron flux variation for those steep energy spectrums.

Xiao et al., 2014], and the effect of oblique chorus $\left(\theta_{m}>15^{\circ}\right)$ has been emphasized in the recent works [e.g., Artemyev et al., 2012; Mourenas et al., 2012, 2014].

The initial PSD is written in the following form

$$
F\left(\alpha_{e}, E_{k}\right)=F_{i}\left(E_{k}\right)\left(\frac{\sin \alpha_{e}}{\sin \alpha_{e i}}\right)^{q},
$$

where $F_{i}\left(E_{k}\right)$ is interpolated from the RBSP-observed PSD for the local pitch angle $\alpha=90^{\circ}$ (the mapped equatorial pitch angle $\alpha_{e}=\alpha_{e i}$ ) at the initial time point $t_{i}$ and $q=2$ is the pitch angle index at $L \approx 6.0$ [see Thorne et al., 2005]. The equivalent extrapolation is applied at the two pitch-angle boundaries $\alpha_{e}=0^{\circ}$ and $90^{\circ}$. The fixed boundary condition is used at $E_{k}=10.0 \mathrm{MeV}$, while a time-varying boundary condition $F=F_{i}\left[1+\frac{t-t_{i}}{t_{e}-t_{i}}\left(\frac{F_{e}}{F_{i}}-1\right)\right]\left(F_{e}\right.$ is the RBSP-observed PSD for the equatorial pitch angle $\alpha_{e}=\alpha_{e i}$ at the final time point $t_{e}$ ) is adopted at $E_{k}=0.1 \mathrm{MeV}$. The electrons with energies near $100 \mathrm{keV}$ can be accelerated to MeV electrons by chorus waves [Horne and Thorne, 1998; Summers et al., 1998], and the lower energy $(<100 \mathrm{keV})$ electrons can contribute to the excitation of chorus waves [e.g., Li et al., 2010; Su et al., 2014a]. For this rapid evolution event, the time step is set to be $2 \mathrm{~s}$.

The comparison between RBSP-observed and STEERB-simulated differential flux spectrums $j=p^{2} F$ for a fixed equatorial pitch angle $\alpha_{e}=40^{\circ}$ at $L^{*}=5.5$ is presented in Figure 8a. Three colors correspond to the three time points denoted by the vertical dashed lines in Figure 3. The local pitch angle is $\alpha \approx 90^{\circ}$ at the first two time points (with the latitude $\lambda \approx 18^{\circ}-19^{\circ}$ ) but $\alpha \approx 40^{\circ}$ at the third time point (with the latitude $\lambda \approx 0^{\circ}$ ). Before the substorm injection, the position $L^{*}=5.5$ was actually beyond the radiation belt outer boundary with a quite soft energy spectrum $j_{\text {pre }}$ (blue). The simulation is initialized soon after the substorm injection with a significantly harder energy spectrum $j_{s}$ (cyan) and ended after about $6 \mathrm{~h}$ yielding the hardest energy spectrum $j_{\mathrm{sc}}$ (red). Reasonable agreement between simulation and observation can be found at the final time point, suggesting that the modeled chorus waves are adequate to explain the "required" 

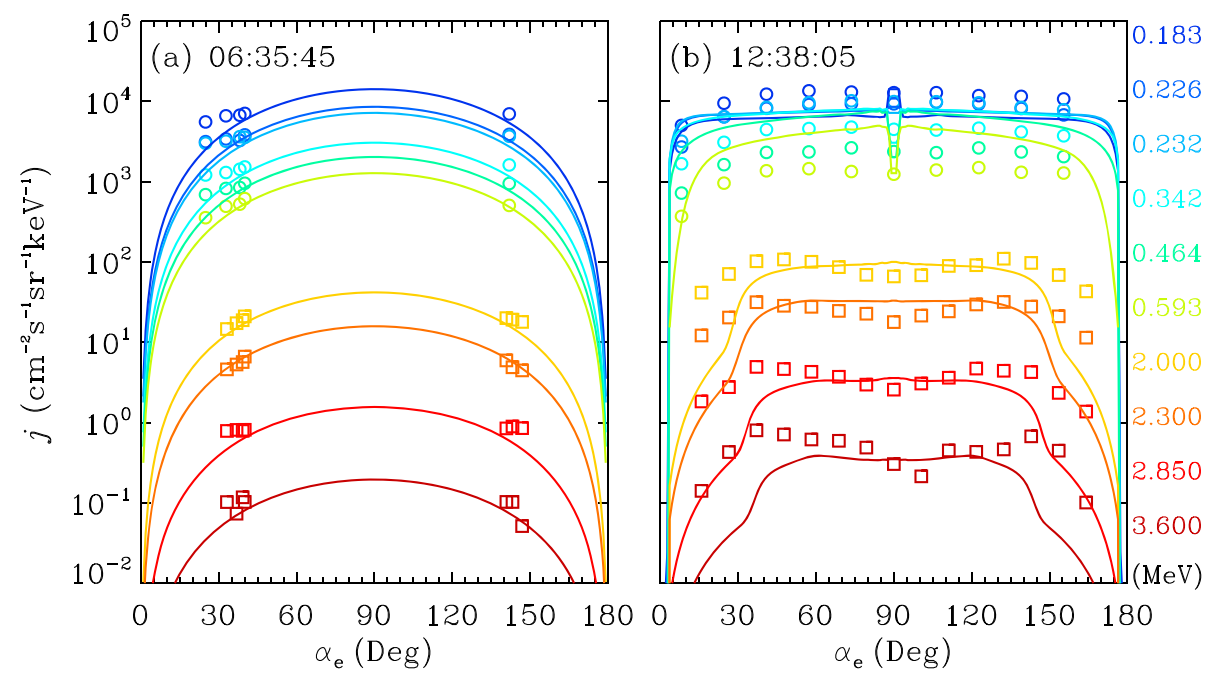

Figure 9. Observed (symbols) and simulated (lines) equatorial pitch angle-dependent differential fluxes $j=p^{2} F$ at two time points. Note that the circles and squares represent the observations of MagEIS and REPT instruments.

electron acceleration after substorm injection. It should be mentioned that the simulation indeed shows the deviation more or less from the observations (for example, at energies around 0.2 MeV and 3.6 MeV), probably caused by the inaccuracy of wave model constructed based on limited observations. Figure $8 \mathrm{~b}$ gives the contribution percentages of substorm injection and chorus waves to the whole event, which are directly calculated as follows:

$$
\begin{aligned}
& P_{s}= \begin{cases}\frac{j_{s}-j_{\text {pre }}}{j_{s c}-j_{\text {pre }}}, & \text { when } j_{s} \leq j_{s c}, \\
1, & \text { when } j_{s}>j_{s c},\end{cases} \\
& P_{c}= \begin{cases}\frac{j_{s c}-j_{s}}{j_{s c}-j_{\text {pre }},} & \text { when } j_{s} \leq j_{s c}, \\
0, & \text { when } j_{s}>j_{s c} .\end{cases}
\end{aligned}
$$

The substorm injection alone can explain the electron flux enhancement at the low energies $(<0.2 \mathrm{MeV})$. With the energy increasing from $0.2 \mathrm{MeV}$ to $5.0 \mathrm{MeV}$, the contribution percentage of substorm injection decreased from $40 \%$ to $20 \%$, while the contribution percentage of chorus waves increased from $60 \%$ to $80 \%$.

The simulations here are implemented at a fixed drift shell $L^{*}$. In fact, the pitch angle distributions at a fixed $L^{*}$ is not observable due to the drift shell splitting in an asymmetrical geomagnetic field [Horne et al., 2003]. A "nonrigorous" comparison between the observed and simulated electron pitch angle distributions at two different time points (denoted by the vertical dashed lines in Figures $3 \mathrm{~b}$ and $3 \mathrm{c}$ ) is presented in Figure 9. The electrons with $\alpha=90^{\circ}$ at 06:35:45 UT and with $\alpha=40^{\circ}$ at 12:38:05 UT were in the same drift shell $L^{*}=5.5$, but the other electrons drifted in some different shells (depending on pitch angle and time). At 06:35:45 UT, the observation data were detected in the off-equatorial region $\left(\lambda \approx 18^{\circ}\right)$, and the local pitch angles $\alpha$ are mapped to the equatorial pitch angle $\alpha_{e}$ through the conservation of magnetic moment $\sin \alpha_{e}=\sin \alpha \cdot\left(B_{e} / B\right)^{1 / 2}$ with the equatorial $B_{e}$ and local $B$ magnetic field strength. Specifically, the local pitch angle $\alpha=90^{\circ}$ approximately corresponds to the equatorial pitch angle $\alpha_{e}=40^{\circ}$ (i.e., $\left(B_{e} / B\right)^{1 / 2} \approx \sin 40^{\circ}$ ). Many observation data gaps are found to occur in the pitch angle direction, and these available observations are generally consistent with the simulations. At 12:38:05 UT, the observation data were collected in the near-equatorial region $\left(\lambda \approx 0.5^{\circ}\right)$, and the local pitch angles $\alpha$ are assumed to equal the equatorial pitch angle $\alpha_{e}$ without any mapping. For the low-energy electrons, the observed flat pitch angle distributions are approximately reproduced by the simulations except around $\alpha_{e}=90^{\circ}$. Those bump or trough structures around $\alpha_{e}=90^{\circ}$ correspond to the region with nearly zero diffusion coefficients (see Figure 7). Other physical processes, e.g., bounce resonance [Shprits, 2009], are required to diffuse the equatorially trapped electrons. For the high-energy electrons, the simulations generally reproduce the observations at the large pitch angles $\left(\alpha_{e}>35^{\circ}\right)$ but obviously underestimate the flux enhancement at the small pitch angles $\left(\alpha_{e}<35^{\circ}\right)$. Such disagreement may be explained by the adoption of dipole field for diffusion coefficient calculation and/or the absence of dayside chorus waves. The quasi-linear calculations of Orlova and Shprits 

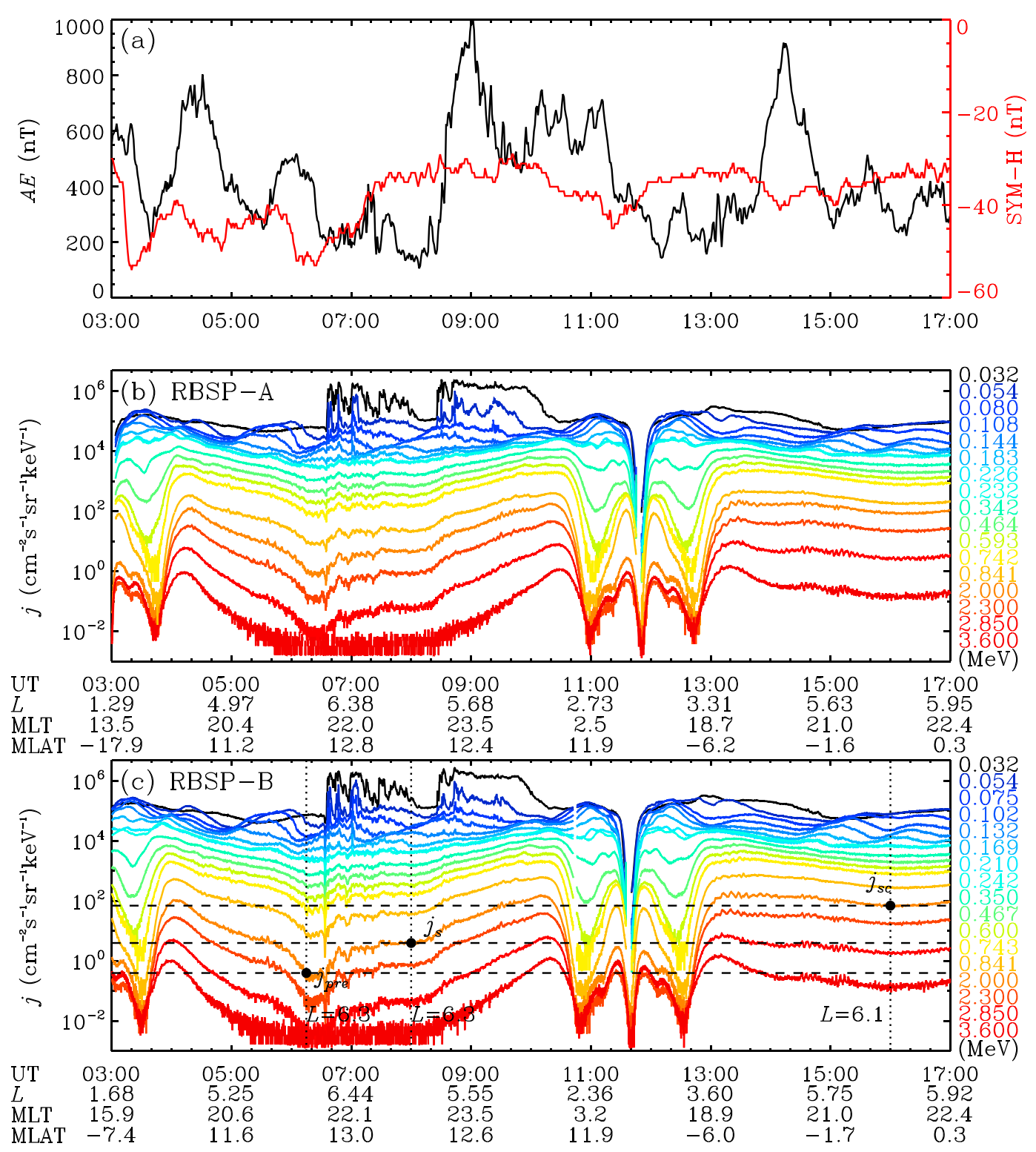

Figure 10. (a) Geomagnetic activity indices $S Y M-H$ and $A E$ and spin-averaged electron fluxes $j$ observed by (b) RBSP-A and (c) RBSP-B for the 2 June 2013 event. Note that the black dots denote the $2 \mathrm{MeV}$ electron differential fluxes at three time points.

[2010] have illustrated that the nightside chorus in the nondipole magnetic field can produce more effective diffusion of electrons with small pitch angles than that in the dipole field. As shown in many quasi-linear simulations [Li et al., 2007; Su et al., 2009, 2011 b; Xiao et al., 2009], the dayside chorus waves alone cannot effectively energize the electrons but can diffuse the electrons from the large pitch angles toward the loss cone. Note that the specific orbits of RBSP and THEMIS satellites during this event did not allow the direct observations of dayside chorus waves (usually distributed in the midlatitude region $\lambda=15^{\circ}-30^{\circ}$ [Horne et al., 2005a]).

Here we reiterate that the contributions of substorm injection and chorus waves are quantified near the outer boundary of radiation belt $\left(L^{*}=5.5\right.$ and $L \approx 6.0$ ). Obviously, in the inner $L^{*}$ region, these contribution percentages can change to some extent. We calculate the Roederer $L^{*}$ based on a geomagnetic field model with the IGRF internal field and the OP77 external field. This field model may not accurately describe the geomagnetic field during the substorm. The fixed $L^{*}$ perhaps corresponds to the different spatial location $L$ in a more realistic geomagnetic field model. However, due to the smooth behavior of the electron fluxes 


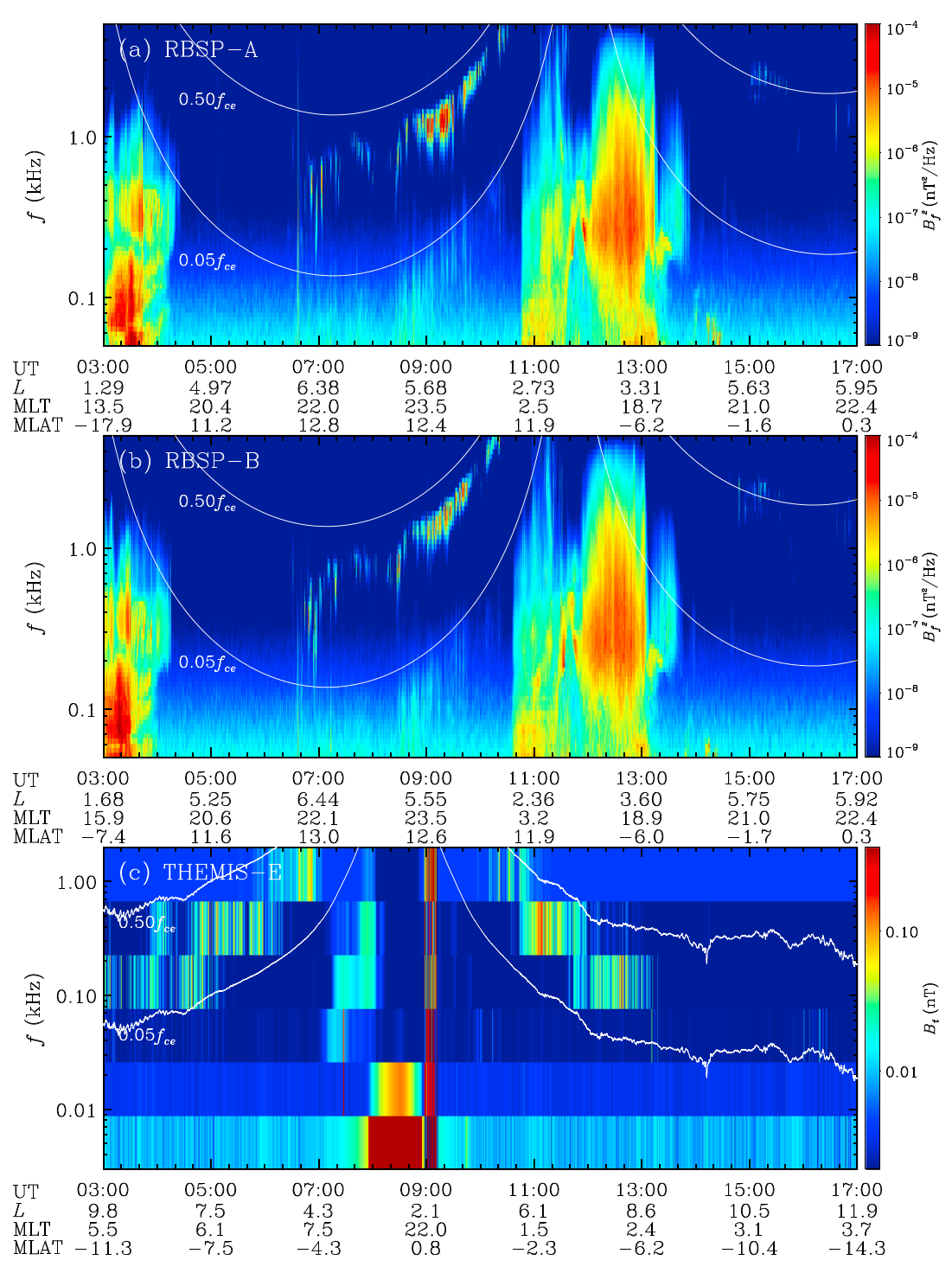

Figure 11. The same as Figure 4 except for the 2 June 2013 event.

around the last two time points (see Figure 3), the application of other geomagnetic field models would not significantly change those contribution percentages.

\subsection{Events on 2 and 29 June 2013}

Figures 10 and 11 show the geomagnetic activity indices, electron fluxes, and VLF/ELF waves for the 2 June 2013 event. There were prolonged substorm and weak storm activities (with maximum $A E=1000 \mathrm{nT}$ and minimum SYM-H $=-55 \mathrm{nT}$ ) throughout this event. The initial outer boundary of radiation belt was around $L=6.3$, detected by RBSP-B at 06:15 UT. The first substorm injection was observed by the twin RBSP satellites around 06:35 UT $(L=6.5)$. After the first substorm injection, the electron fluxes showed obvious enhancement over a wide energy range (Figures $10 \mathrm{~b}$ and 10c), and the chorus waves were excited in the nightside region (Figures 11a and 11b). The second substorm injection was observed around 08:25 UT $(L=6.0)$, causing the noticeable enhancement in both the low-energy electron fluxes and the chorus wave activities. Based on the observations of RBSP-B at 06:15 UT, 08:00 UT, and 16:00 UT, we estimate the contribution percentages of substorm injections and chorus waves to the flux enhancement at $L=6.3$. The electron flux spectrum hardened by chorus waves at $L=6.3$ was not available due to the limited orbital coverage, which is assumed to equal that at $L=6.0$ in order to allow the calculation. At the low energies, the substorm injections can still fully account for the electron flux enhancement. At the high energies, for example, at $E_{k}=2.0 \mathrm{MeV}$, the first substorm injection yielded $P_{s}=\left(j_{s}-j_{\text {pre }}\right) /\left(j_{\mathrm{sc}}-j_{\text {pre }}\right)=6 \%$ of the total flux 

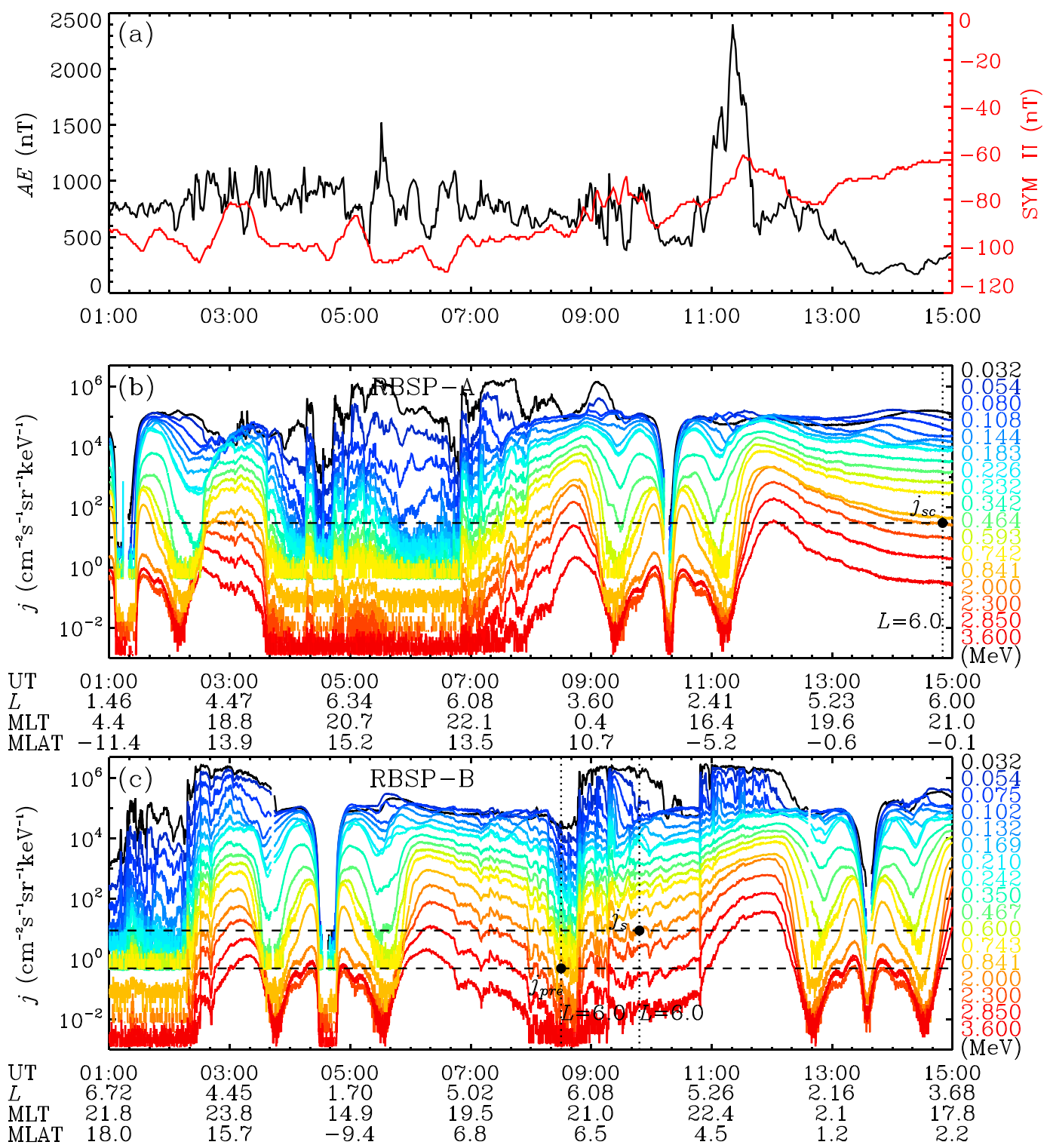

Figure 12. The same as Figure 10 except for the 29 June 2013 event.

enhancement, and the chorus waves can be expected to produce $P_{c}=\left(j_{s c}-j_{s}\right) /\left(j_{s c}-j_{\text {pre }}\right)=94 \%$ of the total flux enhancement (ignoring the effect of the second substorm injection on the high-energy electron fluxes). This contribution percentage of chorus waves was larger than that in the 26 May 2013 event, which may be partially explained by the extended chorus acceleration time $(8 \mathrm{~h})$ in the present event.

Figures 12 and 13 show the geomagnetic activity indices, electron fluxes, and VLF/ELF waves for the 29 June 2013 event. Different from the previous two events, this event had strong substorm and storm activities (with maximum $A E=2400 \mathrm{nT}$ and minimum SYM- $H=-110 \mathrm{nT}$ ). The initial outer boundary of radiation belt was inside $L=5.5$, as observed by RBSP-B before 02:20 UT. The first substorm injection around 02:20 UT largely increased the electron fluxes over a wide energy range (Figure 12c) and simultaneously excited the strong chorus waves (Figure 13b). This substorm injection did not completely fill the spatial region $L>5.5$, as observed by the RBSP-A satellite at 04:00 UT. In the following time period, multiple substorm injections occurred, for example, around 06:50 UT (RBSP-A), 08:50 UT (RBSP-B), and 10:50 UT (RBSP-B). The final outer boundary moved beyond $L=6.0$, as observed by RBSP-A at 14:50 UT. Here we simply estimate the contributions of substorm injections and chorus waves at $L=6.0$ based on the observations at 08:30 UT (RBSP-B), 09:48 UT (RBSP-B), and 14:50 UT (RBSP-A). At the low 


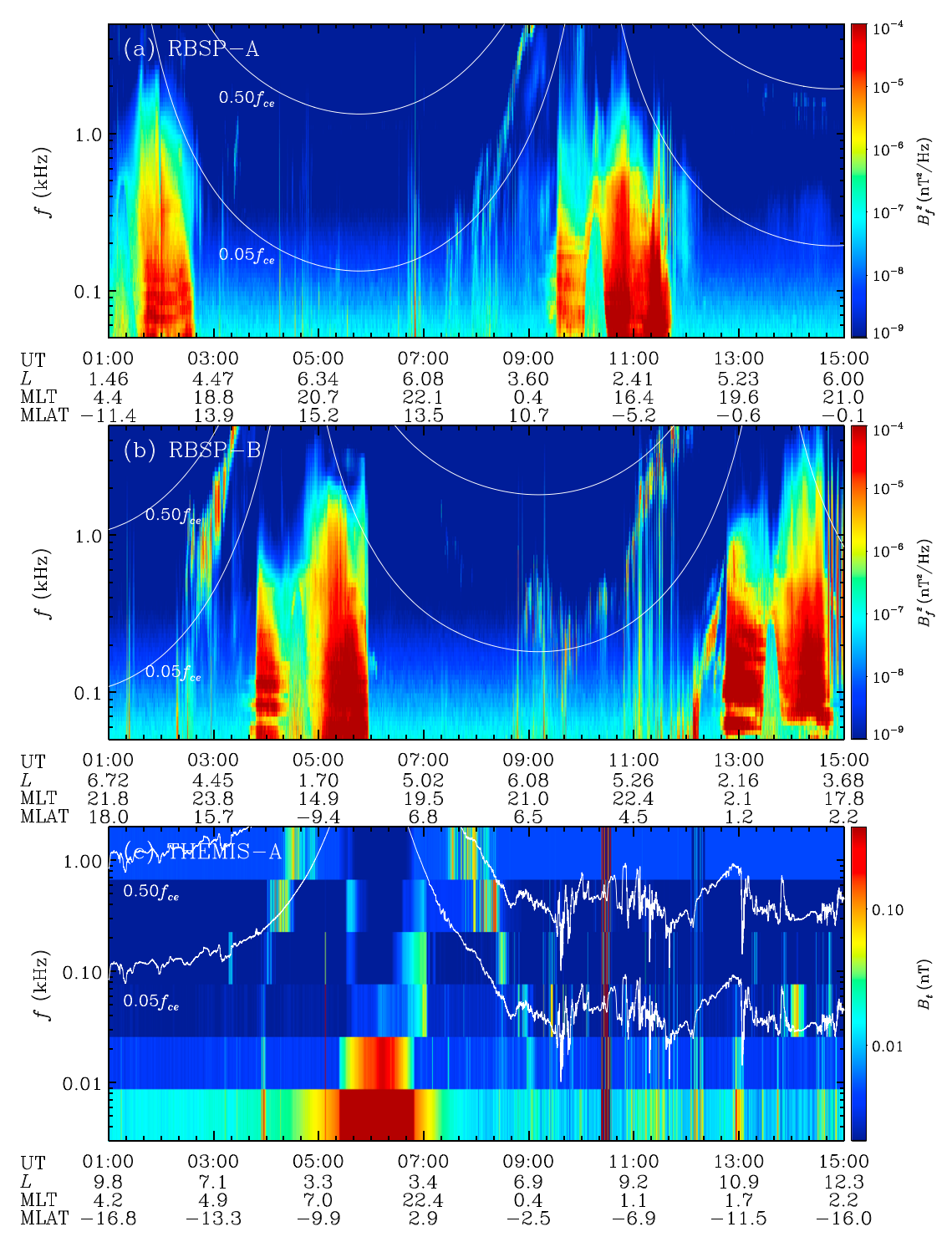

Figure 13. The same as Figure 4 except for the 29 June 2013 event.

energies, the substorm injections were sufficient to explain the flux enhancement. At the high energies, for example, at $E_{k}=2.0 \mathrm{MeV}$, the contribution percentage of the substorm injections is approximately calculated as $P_{s} \approx\left(2 j_{s}-j_{\text {pre }}\right) /\left(j_{\text {sc }}-j_{\text {pre }}\right)=60 \%$ (where the ratio 2 is multiplied considering the $\sim 2$ times flux enhancement caused by the substorm injection at 10:50 UT), and the contribution percentage of chorus waves is roughly considered to be $P_{c} \approx\left(j_{s c}-2 j_{s}\right) /\left(j_{s c}-j_{\text {pre }}\right)=40 \%$. The chorus waves contributed less to the flux enhancement during the present event (compared to those in the 26 May 2013 event), which is reasonable in view of the superposition of multiple substorm injections and the shortening of chorus acceleration time (5 h).

In fact, the radiation belt dynamics in the last two events (involving multiple substorm injections) were much more complex than those in the first event. As stated above, the RBSP satellites for the last two events were not in the appropriate position to observe the electron fluxes at all the three required time points (before the substorm injections, following the substorm injections, and after the further acceleration by chorus waves). Considering the complexity of the two events and the imperfection of the observations, the detailed simulations have not been implemented. Some assumptions have been made to calculate the contribution percentages of substorm injections and chorus waves, which appear to be qualitatively comparable to those for the first event. 


\section{Conclusions and Discussions}

Understanding the electron radiation belt evolution on various timescales is important for space weather nowcasting and forecasting. The substorm injection is usually considered to produce the low-energy (up to several hundreds keV) electron flux enhancement on a timescale of minutes [e.g., Reeves et al., 1990; Friedel et al., 1996; Baker et al., 1997, 1998]. The chorus waves are widely believed to account for the local acceleration of relativistic electrons on a timescale of days [e.g., Horne and Thorne, 1998; Summers et al., 1998; Shprits et al., 2006; Fok et al., 2008; Albert et al., 2009; Su et al., 2010; Xiao et al., 2010; Fu et al., 2011]. The growing network of satellites allows an in-depth understanding of these physical processes. Recently, the analyses of the 17 March 2013 event [Baker et al., 2014; Boyd et al., 2014; Foster et al., 2014] have illustrated once again the importance of substorm injection of low-energy seed electrons for the buildup of relativistic electron fluxes. The joint observations by several radially displaced satellites [Dai et al., 2014] have shown the direct injection of relativistic electrons at the geostationary orbit during substorms. The studies on the 9 October 2012 event [Reeves et al., 2013; Thorne et al., 2013] have demonstrated the rapid acceleration of relativistic electrons by chorus waves within 1 day. The follow-on three-dimensional modeling study for this event [Tu et al., 2014] has shown the importance of data-driven low-energy boundary conditions in the radiation belt simulation.

Here we study the rapid outward extension of electron radiation belt on a timescale of several hours and evaluate the respective contributions of substorm injections and chorus waves to the electron flux enhancement near the outer boundary of radiation belt. We select three events observed by the RBSP and THEMIS satellites on 26 May 2013, 2 June 2013, and 29 June 2013. A comprehensive analysis including both observations and simulations is performed for the first event. Within about $6 \mathrm{~h}$, the outer boundary of the electron radiation belt moved from $L=5.5$ to $L>6.07$. At $L=6.0$, the electron fluxes in the energy range $30 \mathrm{keV}$ to $5 \mathrm{MeV}$ increased by up to 4 orders of magnitude. The substorm injection directly filled the spatial region $L=5.5-6.3$ with electrons over a wide energy range on a timescale of minutes, which were sequentially captured by the two radially displaced RBSP satellites. Following the substorm injection, the strong chorus waves were continuously detected by the RBSP and/or THEMIS satellites. These chorus waves further hardened the injected electron spectrums within about $6 \mathrm{~h}$, which are well reproduced by the data-driven STEERB simulations. For the whole event, the flux enhancement at the low energies $(<0.2 \mathrm{MeV})$ can be fully attributed to the substorm injection, while the flux enhancement at the high energies (0.2-5.0 $\mathrm{MeV}$ ) can be produced by the combination of substorm injection (contribution percentage $20 \%-40 \%$ ) and chorus waves (contribution percentage 60\%-80\%). Some simple analyses are made for the other two events. The corresponding contributions of substorm injections and chorus waves were qualitatively comparable to those in the first event. The quantitative differences in those contribution percentages may be caused by the extending/shortening of chorus acceleration time or the superposition of multiple substorm injections.

\section{References}

Agapitov, O., A. Artemyev, V. Krasnoselskikh, Y. V. Khotyaintsev, D. Mourenas, H. Breuillard, M. Balikhin, and G. Rolland (2013), Statistics of whistler mode waves in the outer radiation belt: Cluster STAFF-SA measurements, J. Geophys. Res. Space Physics, 118, 3407-3420, doi:10.1002/jgra.50312.

Albert, J. M., N. P. Meredith, and R. B. Horne (2009), Three-dimensional diffusion simulation of outer radiation belt electrons during the October 9, 1990, magnetic storm, J. Geophys. Res., 114, A09214, doi:10.1029/2009JA014336.

Angelopoulos, V. (2008), The THEMIS mission, Space Sci. Rev., 141, 5-34, doi:10.1007/s11214-008-9336-1.

Artemyev, A., O. Agapitov, H. Breuillard, V. Krasnoselskikh, and G. Rolland (2012), Electron pitch-angle diffusion in radiation belts: The effects of whistler wave oblique propagation, Geophys. Res. Lett., 39, L08105, doi:10.1029/2012GL051393.

Baker, D. N., et al. (1997), Recurrent geomagnetic storms and relativistic electron enhancements in the outer magnetosphere: ISTP coordinated measurements, J. Geophys. Res., 102, 14,141-14,148, doi:10.1029/97JA00565.

Baker, D. N., T. I. Pulkkinen, X. Li, S. G. Kanekal, J. B. Blake, R. S. Selesnick, M. G. Henderson, G. D. Reeves, H. E. Spence, and G. Rostoker (1998), Coronal mass ejections, magnetic clouds, and relativistic magnetospheric electron events: ISTP, J. Geophys. Res., 103, 17,279-17,292, doi:10.1029/97JA03329.

Baker, D. N., et al. (2013a), The Relativistic Electron-Proton Telescope (REPT) instrument on board the Radiation Belt Storm Probes (RBSP) spacecraft: Characterization of Earth's radiation belt high-energy particle populations, Space Sci. Rev., 179, 337-381, doi:10.1007/s11214-012-9950-9.

Baker, D. N., et al. (2013b), A long-lived relativistic electron storage ring embedded in Earth's outer Van Allen belt, Science, 340(6129), 186-190.

Baker, D. N., et al. (2014), Gradual diffusion and punctuated phase space density enhancements of highly relativistic electrons: Van Allen Probes observations, Geophys. Res. Lett., 41, 1351-1358, doi:10.1002/2013GL058942.

Blake, J. B., W. A. Kolasinski, R. W. Fillius, and E. G. Mullen (1992), Injection of electrons and protons with energies of tens of MeV into L less than 3 on 24 March 1991, Geophys. Res. Lett., 19, 821-824, doi:10.1029/92GL00624.

Blake, J. B., et al. (2013), The Magnetic Electron Ion Spectrometer (MagEIS) instruments aboard the Radiation Belt Storm Probes (RBSP) spacecraft, Space Sci. Rev., 179, 383-421, doi:10.1007/s11214-013-9991-8. 
Bonnell, J. W., F. S. Mozer, G. T. Delory, A. J. Hull, R. E. Ergun, C. M. Cully, V. Angelopoulos, and P. R. Harvey (2008), The Electric Field Instrument (EFI) for THEMIS, Space Sci. Rev., 141, 303-341, doi:10.1007/s11214-008-9469-2.

Boyd, A. J., H. E. Spence, S. G. Claudepierre, J. F. Fennell, J. B. Blake, D. N. Baker, G. D. Reeves, and D. L. Turner (2014), Quantifying the radiation belt seed population in the 17 March 2013 electron acceleration event, Geophys. Res. Lett., 41, 2275-2281, doi:10.1002/2014GL059626.

Brautigam, D. H., and J. M. Albert (2000), Radial diffusion analysis of outer radiation belt electrons during the October 9, 1990, magnetic storm, J. Geophys. Res., 105, 291-310, doi:10.1029/1999JA900344.

Cattell, C., et al. (2008), Discovery of very large amplitude whistler-mode waves in Earth's radiation belts, Geophys. Res. Lett., 35, L01105, doi:10.1029/2007GL032009.

Dai, L., J. R. Wygant, C. A. Cattell, S. Thaller, K. Kersten, A. Breneman, X. Tang, R. H. Friedel, S. G. Claudepierre, and X. Tao (2014), Evidence for injection of relativistic electrons into the Earth's outer radiation belt via intense substorm electric fields, Geophys. Res. Lett., 41 , 1133-1141, doi:10.1002/2014GL059228.

Fok, M.-C., R. B. Horne, N. P. Meredith, and S. A. Glauert (2008), Radiation Belt Environment model: Application to space weather nowcasting, J. Geophys. Res., 113, A03S08, doi:10.1029/2007JA012558.

Foster, J. C., et al. (2014), Prompt energization of relativistic and highly relativistic electrons during a substorm interval: Van Allen Probes observations, Geophys. Res. Lett., 41, 20-25, doi:10.1002/2013GL058438.

Friedel, R. H. W., A. Korth, and G. Kremser (1996), Substorm onsets observed by CRRES: Determination of energetic particle source regions, J. Geophys. Res., 101, 13,137-13,154, doi:10.1029/96JA00399.

Fu, H. S., J. B. Cao, B. Yang, and H. Y. Lu (2011), Electron loss and acceleration during storm time: The contribution of wave-particle interaction, radial diffusion, and transport processes, J. Geophys. Res., 116, A10210, doi:10.1029/2011JA016672.

Glauert, S. A., and R. B. Horne (2005), Calculation of pitch angle and energy diffusion coefficients with the PADIE code, J. Geophys. Res., 110, A04206, doi:10.1029/2004JA010851.

Glauert, S. A., R. B. Horne, and N. P. Meredith (2014), Three-dimensional electron radiation belt simulations using the BAS Radiation Belt Model with new diffusion models for chorus, plasmaspheric hiss, and lightning-generated whistlers, J. Geophys. Res. Space Physics, 119, 268-289, doi:10.1002/2013JA019281.

Grinsted, A., J. C. Moore, and S. Jevrejeva (2004), Application of the cross wavelet transform and wavelet coherence to geophysical time series, Nonlinear Processes Geophys., 11, 561-566.

Horne, R. B., and R. M. Thorne (1998), Potential waves for relativistic electron scattering and stochastic acceleration during magnetic storms, Geophys. Res. Lett., 25, 3011-3014, doi:10.1029/98GL01002.

Horne, R. B., N. P. Meredith, R. M. Thorne, D. Heynderickx, R. H. A. Iles, and R. R. Anderson (2003), Evolution of energetic electron pitch angle distributions during storm time electron acceleration to megaelectronvolt energies, J. Geophys. Res., 108(A1), 1016, doi:10.1029/2001JA009165.

Horne, R. B., R. M. Thorne, S. A. Glauert, J. M. Albert, N. P. Meredith, and R. R. Anderson (2005a), Timescale for radiation belt electron acceleration by whistler mode chorus waves, J. Geophys. Res., 110, A03225, doi:10.1029/2004JA010811.

Horne, R. B., et al. (2005b), Wave acceleration of electrons in the Van Allen radiation belts, Nature, 437, 227-230, doi:10.1038/nature03939.

Hudson, M. K., S. R. Elkington, J. G. Lyon, V. A. Marchenko, I. Roth, M. Temerin, J. B. Blake, M. S. Gussenhoven, and J. R. Wygant (1997), Simulations of radiation belt formation during storm sudden commencements, J. Geophys. Res., 102, 14,087-14,102, doi:10.1029/97JA03995.

Hwang, J., K. W. Min, E. Lee, C. Lee, and D. Y. Lee (2004), A case study to determine the relationship of relativistic electron events to substorm injections and ULF power, Geophys. Res. Lett., 31, L23801, doi:10.1029/2004GL021544.

Kletzing, C. A., et al. (2013), The Electric and Magnetic Field Instrument Suite and Integrated Science (EMFISIS) on RBSP, Space Sci. Rev., 179, 127-181, doi:10.1007/s11214-013-9993-6.

Li, L., J. Cao, and G. Zhou (2005), Combined acceleration of electrons by whistler-mode and compressional ULF turbulences near the geosynchronous orbit, J. Geophys. Res., 110, A03203, doi:10.1029/2004JA010628.

Li, W., Y. Y. Shprits, and R. M. Thorne (2007), Dynamic evolution of energetic outer zone electrons due to wave-particle interactions during storms, J. Geophys. Res., 112, A10220, doi:10.1029/2007JA012368.

Li, W., R. M. Thorne, V. Angelopoulos, J. W. Bonnell, J. P. McFadden, C. W. Carlson, O. LeContel, A. Roux, K. H. Glassmeier, and H. U. Auster (2009), Evaluation of whistler-mode chorus intensification on the nightside during an injection event observed on the THEMIS spacecraft, J. Geophys. Res., 114, A00C14, doi:10.1029/2008JA013554.

Li, W., et al. (2010), THEMIS analysis of observed equatorial electron distributions responsible for the chorus excitation, J. Geophys. Res., 115, A00F11, doi:10.1029/2009JA014845.

Li, W., et al. (2014), Radiation belt electron acceleration by chorus waves during the 17 March 2013 storm, J. Geophys. Res. Space Physics, 119, 4681-4693, doi:10.1002/2014JA019945.

Li, X., D. N. Baker, M. Temerin, G. D. Reeves, and R. D. Belian (1998), Simulation of dispersionless injections and drift echoes of energetic electrons associated with substorms, Geophys. Res. Lett., 25, 3763-3766, doi:10.1029/1998GL900001.

Li, X., D. N. Baker, S. G. Kanekal, M. Looper, and M. Temerin (2001), Long term measurements of radiation belts by SAMPEX and their variations, Geophys. Res. Lett., 28, 3827-3830, doi:10.1029/2001GL013586.

Li, X., T. E. Sarris, D. N. Baker, W. K. Peterson, and H. J. Singer (2003), Simulation of energetic particle injections associated with a substorm on August 27, 2001, Geophys. Res. Lett., 30(1), 1004, doi:10.1029/2002GL015967.

Lui, A. T. Y., Q.-G. Zong, C. Wang, and M. W. Dunlop (2012), Electron source associated with dipolarization at the outer boundary of the radiation belts: Non-storm cases, J. Geophys. Res., 117, A10224, doi:10.1029/2012JA018084.

Liu, S., M. W. Chen, L. R. Lyons, H. Korth, J. M. Albert, J. L. Roeder, P. C. Anderson, and M. F. Thomsen (2003), Contribution of convective transport to stormtime ring current electron injection, J. Geophys. Res., 108(A10), 1372, doi:10.1029/2003JA010004.

Mauk, B. H., N. J. Fox, S. G. Kanekal, R. L. Kessel, D. G. Sibeck, and A. Ukhorskiy (2013), Science objectives and rationale for the Radiation Belt Storm Probes mission, Space Sci. Rev., 179, 3-27, doi:10.1007/s11214-012-9908-y.

Mcllwain, C. E. (1974), Substorm injection boundaries, in Magnetospheric Physics, edited by B. M. McCormac, pp. 143-154, Springer, New York.

Meredith, N. P., R. B. Horne, and R. R. Anderson (2001), Substorm dependence of chorus amplitudes: Implications for the acceleration of electrons to relativistic energies, J. Geophys. Res., 106, 13,165-13,178, doi:10.1029/2000JA900156.

Meredith, N. P., R. B. Horne, A. Sicard-Piet, D. Boscher, K. H. Yearby, W. Li, and R. M. Thorne (2012), Global model of lower band and upper band chorus from multiple satellite observations, J. Geophys. Res., 117, A10225, doi:10.1029/2012JA017978.

Miyoshi, Y., A. Morioka, H. Misawa, T. Obara, T. Nagai, and Y. Kasahara (2003), Rebuilding process of the outer radiation belt during the 3 November 1993 magnetic storm: NOAA and Exos-D observations, J. Geophys. Res., 108(A1), 1004, doi:10.1029/2001 JA007542. 
Moore, T. E., R. L. Arnoldy, J. Feynman, and D. A. Hardy (1981), Propagating substorm injection fronts, J. Geophys. Res., 86, 6713-6726, doi:10.1029/JA086iA08p06713.

Mourenas, D., A. Artemyev, O. Agapitov, and V. Krasnoselskikh (2012), Acceleration of radiation belts electrons by oblique chorus waves, J. Geophys. Res., 117, A10212, doi:10.1029/2012JA018041.

Mourenas, D., A. V. Artemyev, O. V. Agapitov, and V. Krasnoselskikh (2014), Consequences of geomagnetic activity on energization and loss of radiation belt electrons by oblique chorus waves, J. Geophys. Res. Space Physics, 119, 2775-2796, doi:10.1002/2013JA019674.

Olson, W. P., and K. A. Pfitzer (1982), A dynamic model of the magnetospheric magnetic and electric fields for July 29, 1977, J. Geophys. Res., 87, 5943-5948, doi:10.1029/JA087iA08p05943.

Omura, Y., Y. Katoh, and D. Summers (2008), Theory and simulation of the generation of whistler-mode chorus, J. Geophys. Res., 113, A04223, doi:10.1029/2007JA012622.

Orlova, K. G., and Y. Y. Shprits (2010), Dependence of pitch-angle scattering rates and loss timescales on the magnetic field model, Geophys. Res. Lett., 37, L05105, doi:10.1029/2009GL041639.

Reeves, G. D., T. A. Fritz, T. E. Cayton, and R. D. Belian (1990), Multi-satellite measurements of the substorm injection region, Geophys. Res. Lett., 17, 2015-2018, doi:10.1029/GL017i011p02015.

Reeves, G. D., D. N. Baker, R. D. Belian, J. B. Blake, T. E. Cayton, J. F. Fennell, R. H. W. Friedel, M. M. Meier, R. S. Selesnick, and H. E. Spence (1998), The global response of relativistic radiation belt electrons to the January 1997 magnetic cloud, Geophys. Res. Lett., 25, 3265-3268, doi:10.1029/98GL02509.

Reeves, G. D., K. L. McAdams, R. H. W. Friedel, and T. P. O'Brien (2003), Acceleration and loss of relativistic electrons during geomagnetic storms, Geophys. Res. Lett., 30(10), 1529, doi:10.1029/2002GL016513.

Reeves, G. D., et al. (2013), Electron acceleration in the heart of the Van Allen radiation belts, Science, 341(6149), 991-994.

Russell, C. T., and R. L. McPherron (1973), The magnetotail and substorms, Space Sci. Rev., 15, 205-266, doi:10.1007/BF00169321.

Santolík, O., D. A. Gurnett, J. S. Pickett, M. Parrot, and N. Cornilleau-Wehrlin (2003a), Spatio-temporal structure of storm-time chorus, J. Geophys. Res., 108(A7), 1278, doi:10.1029/2002JA009791.

Santolík, O., M. Parrot, and F. Lefeuvre (2003b), Singular value decomposition methods for wave propagation analysis, Radio Sci., 38(1), 1010, doi:10.1029/2000RS002523.

Santolík, O., C. A. Kletzing, W. S. Kurth, G. B. Hospodarsky, and S. R. Bounds (2014a), Fine structure of large-amplitude chorus wave packets, Geophys. Res. Lett., 41, 293-299, doi:10.1002/2013GL058889.

Santolík, O., E. Macúšová, I. Kolmašová, N. Cornilleau-Wehrlin, and Y. Conchy (2014b), Propagation of lower-band whistler-mode waves in the outer Van Allen belt: Systematic analysis of 11 years of multi-component data from the Cluster spacecraft, Geophys. Res. Lett., 41, 2729-2737, doi:10.1002/2014GL059815.

Selesnick, R. S., J. B. Blake, and R. A. Mewaldt (2003), Atmospheric losses of radiation belt electrons, J. Geophys. Res., 108(A12), 1468, doi:10.1029/2003JA010160.

Sheeley, B. W., M. B. Moldwin, H. K. Rassoul, and R. R. Anderson (2001), An empirical plasmasphere and trough density model: CRRES observations, J. Geophys. Res., 106, 25,631-25,642, doi:10.1029/2000JA000286.

Shprits, Y. Y. (2009), Potential waves for pitch-angle scattering of near-equatorially mirroring energetic electrons due to the violation of the second adiabatic invariant, Geophys. Res. Lett., 36, L12106, doi:10.1029/2009GL038322.

Shprits, Y. Y., R. M. Thorne, R. B. Horne, S. A. Glauert, M. Cartwright, C. T. Russell, D. N. Baker, and S. G. Kanekal (2006), Acceleration mechanism responsible for the formation of the new radiation belt during the 2003 Halloween solar storm, Geophys. Res. Lett., 33 , L05104, doi:10.1029/2005GL024256.

Shprits, Y. Y., L. Chen, and R. M. Thorne (2009a), Simulations of pitch angle scattering of relativistic electrons with MLT-dependent diffusion coefficients, J. Geophys. Res., 114, A03219, doi:10.1029/2008JA013695.

Shprits, Y. Y., D. Subbotin, and B. Ni (2009b), Evolution of electron fluxes in the outer radiation belt computed with the VERB code, J. Geophys. Res., 114, A11209, doi:10.1029/2008JA013784.

Shprits, Y. Y., D. Subbotin, A. Drozdov, M. E. Usanova, A. Kellerman, K. Orlova, D. N. Baker, D. L. Turner, and K.-C. Kim (2013), Unusual stable trapping of the ultra-relativistic electrons in the Van Allen radiation belts, Nat. Phys., 9, 699-703, doi:10.1038/nphys2760.

Spence, H. E., et al. (2013), Science goals and overview of the Energetic Particle, Composition, and Thermal Plasma (ECT) suite on NASA's Radiation Belt Storm Probes (RBSP) mission, Space Sci. Rev., 179, 311-336, doi:10.1007/s11214-013-0007-5.

Stern, D. P. (1973), A study of the electric field in an open magnetospheric model, J. Geophys. Res., 78, 7292-7305, doi:10.1029/JA078i031p07292.

$\mathrm{Su}, \mathrm{Z} ., \mathrm{H}$. Zheng, and S. Wang (2009), Dynamic evolution of energetic outer zone electrons due to whistler-mode chorus based on a realistic density model, J. Geophys. Res., 114, A07201, doi:10.1029/2008JA014013.

$\mathrm{Su}, \mathrm{Z} .$, F. Xiao, H. Zheng, and S. Wang (2010), STEERB: A three-dimensional code for storm-time evolution of electron radiation belt, J. Geophys. Res., 115, A09208, doi:10.1029/2009JA015210.

Su, Z., F. Xiao, H. Zheng, and S. Wang (2011a), CRRES observation and STEERB simulation of the 9 October 1990 electron radiation belt dropout event, Geophys. Res. Lett., 38, L06106, doi:10.1029/2011GL046873.

$\mathrm{Su}, \mathrm{Z} .$, H. Zheng, L. Chen, and S. Wang (2011b), Numerical simulations of storm-time outer radiation belt dynamics by wave-particle interactions including cross diffusion, J. Atmos. Sol. Terr. Phys., 73, 95-105, doi:10.1016/j.jastp.2009.08.002.

Su, Z., et al. (2014a), Intense duskside lower band chorus waves observed by Van Allen Probes: Generation and potential acceleration effect on radiation belt electrons, J. Geophys. Res. Space Physics, 119, 4266-4273, doi:10.1002/2014JA019919.

$\mathrm{Su}$, Z., et al. (2014b), Nonstorm time dynamics of electron radiation belts observed by the Van Allen Probes, Geophys. Res. Lett., 41, 229-235, doi:10.1002/2013GL058912.

Summers, D., R. M. Thorne, and F. Xiao (1998), Relativistic theory of wave-particle resonant diffusion with application to electron acceleration in the magnetosphere, J. Geophys. Res., 103, 20,487-20,500.

Summers, D., C. Ma, N. P. Meredith, R. B. Horne, R. M. Thorne, D. Heynderickx, and R. R. Anderson (2002), Model of the energization of outer-zone electrons by whistler-mode chorus during the October 9, 1990 geomagnetic storm, Geophys. Res. Lett., 29(24), 2174, doi:10.1029/2002GL016039.

Thorne, R. M., T. P. O'Brien, Y. Y. Shprits, D. Summers, and R. B. Horne (2005), Timescale for MeV electron microburst loss during geomagnetic storms, J. Geophys. Res., 110, A09202, doi:10.1029/2004JA010882.

Thorne, R. M., et al. (2013), Rapid local acceleration of relativistic radiation-belt electrons by magnetospheric chorus, Nature, 504, 411-414, doi:10.1038/nature 12889.

Tsurutani, B. T., and E. J. Smith (1974), Postmidnight chorus: A substorm phenomenon, J. Geophys. Res., 79, 118-127, doi:10.1029/JA079i001p00118. 
Tu, W., G. S. Cunningham, Y. Chen, S. K. Morley, G. D. Reeves, J. B. Blake, D. N. Baker, and H. Spence (2014), Event-specific chorus wave and electron seed population models in DREAM3D using the Van Allen Probes, Geophys. Res. Lett., 41, 1359-1366, doi:10.1002/2013GL058819.

Volland, H. (1973), A semiempirical model of large-scale magnetospheric electric fields, J. Geophys. Res., 78, 171-180, doi:10.1029/JA078i001p00171.

Xiao, F., Z. Su, H. Zheng, and S. Wang (2009), Modeling of outer radiation belt electrons by multidimensional diffusion process, J. Geophys. Res., 114, A03201, doi:10.1029/2008JA013580.

Xiao, F., Z. Su, H. Zheng, and S. Wang (2010), Three-dimensional simulations of outer radiation belt electron dynamics including cross diffusion terms, J. Geophys. Res., 115, A05216, doi:10.1029/2009JA014541.

Xiao, F., et al. (2014), Chorus acceleration of radiation belt relativistic electrons during March 2013 geomagnetic storm, J. Geophys. Res. Space Physics, 119, 3325-3332, doi:10.1002/2014JA019822.

Zong, Q., X.-Z. Zhou, Y. F. Wang, X. Li, P. Song, D. N. Baker, T. A. Fritz, P. W. Daly, M. Dunlop, and A. Pedersen (2009), Energetic electron response to ULF waves induced by interplanetary shocks in the outer radiation belt, J. Geophys. Res., 114, A10204, doi:10.1029/2009JA014393. 